\title{
loỉ rhéologique incrémentale pour les sols et application par la méthode des éléments finis
}

\section{Marc Boulon}

Ingénieur E.C.P., Maître-Assistant à 1'U.S.M.G.

\section{René Chambon}

Ingénieur E.I.H., Assistant à I'U.S.M.G.

\section{Félix Darve}

Ingénieur E.C.P., Assistant à 1'U.S.M.G.

\section{LOI RHEOLOGIQUE INCREMENTALE POUR LES SOLS \\ ET APPLICATIONS PAR LA METHODE DES ELEMENTS FINIS}

Une loi rhéologique complète, valable pour les milieux granulaires et des applications à des problèmes classiques de mécanique des sols, est présentée.

Cette loi a trait aux matériaux non visqueux. Elle regroupe toutes les descriptions partielles connues des sols et rend compte des charges comme des décharges. Elle est écrite sous forme incrémentale. Ainsi, le comportement du milieu dépend de son histoire. La détermination expérimentale des paramètres représentatifs du matériau est réalisée à partir d'une famille d'essais triaxiaux classiques (en compression et en extension).

Dans un premier temps, on compare les résultats d'intégration de cette loi, sur une vaste gamme de chemins de sollicitation homogènes, avec les mesures correspondantes. Pour cela, on a utilisé des essais réalisés sur des presses tridimensionnelles (appareils triaxiaux "vrais"). La coïncidence s'est avérée satisfaisante, même pour des chemins très différents des chemins triaxaux classiques ayant servi au calcul des paramètres.

Dans un second temps, cette loi est utilisée sous forme simplifiée, dans des programmes de calcul basés sur la méthode des éléments finis en déplacements. Dans le cas d'un matériau analogique bidimensionnel composé de petits rouleaux, les problèmes suivants sont traités :

- simulation d'un triaxial fretté ;

- enfoncement d'une fondation superficielle rigide et rugueuse :

- mur de soutènement (poussée et butée).

\section{INCREMENTAL RHEOLOGICAL LAW FOR SOILS AND APPLICATIONS BY THE FINITE ELEMENTS METHOD}

A complete rheological law, valid for granular media and applications to conventional problems of soil mechanics, is presented.

This law relates to non-viscous materials. It takes in all the known partial descriptions of soils and accounts for both loads and lightenings. It is described in incremental form. Thus, the behaviour of the medium depends on its history. The experimental determination of the representative parameters of the material is effected on the basis of a family of conventional triaxial tests (in compression and in extension).

In a first stage are compared the results of integration of this law, on a vast range of homogeneous stress paths, with the corresponding measurements. For this, tests carried out on threedimensional presses ( «truen triaxial apparatus) have been used. The coincidence proved to be satisfactory, even for paths very different from the conventional axial paths having been used in the computing of the parameters.

In a second stage this law is used in a simplified form, in computing programmes based on the method of finite elements in displacements. In the case of a two-dimensional analogical material composed of small rollers, the following problems are dealt with:

- simulation of a hooped triaxial;

- sinking of a rigid and rough shallow foundation;

- retaining wall (active and passive pressure). 
Comité Français de Mécanique des Sols 


\title{
loi rhéologique incrémentale pour les sols et application par la méthode des éléments finis
}

\author{
par Marc BOULON, René CHAMBON et Félix DARVE
}

\section{INTRODUCTION}

Pour les problèmes de mécanique des sols, il convient de s'intéresser aux déformations comme aux contraintes car la situation d'un ouvrage vis-à-vis de la rupture ne peut être décrite par un seul nombre (coefficient de sécurité), eu égard en particulier au grand nombre de possibilités différentes qu'a l'ouvrage de se rompre. $\mathrm{La}$ détermination de la relation contrainte-déformation (et même plus précisément de la relation histoire des contraintes - histoire des déformations), c'est-à-dire de la loi rhéologique, est donc primordiale pour pouvoir effectuer un calcul en vue de la prévision du comportement d'un ouvrage. Son influence sur les résultats est considérable.

Si nous exceptons la plasticité parfaite qui connaît un renouveau très important avec la théorie des étatslimites (Salençon [1]), nous pouvons classer les lois rhéologiques intergranulaires utilisées pour les calculs de mécanique des sols en quatre grandes catégories selon la schématisation réalisée des propriétés réelles du matériau.

\section{1) L'élasticité linéaire :}

Elle peut fournir des ordres de grandeur pour des problèmes de déformations sous charges de service. Zienkiewicz [2] a été un pionnier en la matière sur le plan européen.

2) Les lois relevant de l'élasticité non linéaire :

Les travaux de Zienkiewicz en Grande-Bretagne, Duncan [3] à Berkeley, Franck [4] et Guellec au Laboratoire Central des Ponts et Chaussées se rattachent à cette classe de lois. S'il est facile d'introduire ces lois dans les programmes de calcul, il est par contre assez difficile de déterminer les caractéristiques mécaniques à prendre en compte. Par ailleurs, elles peuvent conduire non seulement à des erreurs importantes, mais surtout à des comportements aberrants pour des chemins de sollicitation éloignés des chemins de mesure réalisés en laboratoire. En bref, ces lois, comme l'élasticité linéaire, sont partielles, en ce sens qu'elles ne décrivent que certains types de sollicitations.
3) Les lois issues de schémas élastiques-plastiques :

Citons les travaux de Zienkiewicz, Deere [5] (Université de l'Illinois), Whitmann [6] (M.I.T.), Guellec et Ricard [7]. Inattaquables sur le plan théorique, représentant mieux la réalité physique que les précédentes, elles donnent une idée des états limites qui pourraient être néfastes aux ouvrages; mais vu la simplification faite, le problème subsiste quant à la valeur numérique des paramètres à injecter dans les calculs.

4) Les descriptions incrémentales de comportements physiques (théorie de Roscoe [8] à Cambridge par exemple) :

Dans ce type d'écriture, on s'affanchit de tout schéma de comportement, et on cherche à « suivre la nature pas à pas " en formulant une loi tangente respectant les principes de la thermodynamique. Le modèle que nous proposons s'apparente à cette famille : le "calage " de la loi est réalisé sur un réseau de chemins triaxiaux classiques (compression et extension) ; tous les paramètres entrés en données dans les programmes de calcul ont une signification physique claire et ne nécessitent pas d'évaluation a priori. Bien entendu, ce modèle ne prétend pas résoudre tous les problèmes de mécanique des sols, et son utilisation présente encore un certain nombre de difficultés non résolues,

Cette loi est présentée dans la première partie de la présente étude. La seconde partie est consacrée à un contrôle méthodique des résultats d'intégration de la loi sur essais homogènes (comparaison avec des essais réels réalisés sur appareil triaxial vrai). Enfin, la troisième partie consiste en des comparaisons calculexpérience pour des cas réels : il s'agit d'essais triaxiaux frettés, d'essais de plaque et de problèmes de pousséebutée traités sur modèle analogique bidimensionnel (rouleaux de Schneebeli). Ce modèle est le seul sur lequel des observations ponctuelles précises peuvent être faites à l'heure actuelle.

\section{LOI RHEOLOGIQUE INCREMENTALE DES MILIEUX GRANULAIRES : LES PRINCIPALES HYPOTHESES}

Nous nous bornerons ici à dégager les principales hypothèses sur lesquelles notre loi repose. Nous n'entrerons donc dans aucun développement mathématique et tenterons, plutôt, de mettre en avant la " philo-

(*) Conférence présentée devant le Comité Français de la Mécanique des Sols et des Fondations, le 13 octobre 1975. sophie » à la base de notre démarche. Nous utiliserons ainsi fréquemment un schéma monodimensionnel pour soutenir concrètement nos raisonnements; la loi présentée, tridimensionnelle, est évidemment beaucoup plus générale que ce schéma. Pour avoir le détail des raisonnements, le lecteur pourra se référer à Darve [9], 


\subsection{Forme générale de la loi}

Précisons tout d'abord que tous les milieux considérés par la suite sont non-visqueux. Une loi incrémentale est la relation liant alors une petite sollicitation (notée « $d \varepsilon »)$, appliquée à un instant donné à un échantillon homogène, à la petite réponse (notée « $d \sigma »$ ).

En linéarisant la loi rhéologique globale du milieu dans un certain voisinage du point actuel en contraintes et en déformations, nous pouvons écrire :

$d \sigma=\mathrm{M} d \varepsilon$ où $\mathrm{M}$ est un matrice carrée $6 \times 6$.

Sur le schéma monodimensionnel (fig. 1), cette hypothèse reviendrait à relier dans un certain voisinage du point $\left(\mathrm{F}, l-l_{o}\right)$ le petit accroissement de force $d \mathrm{~F}$ à la petite variation de longueur $d l$ par une relation linéaire du type : $d \mathrm{~F}=\mathrm{K} d l$ en assimilant ainsi la courbe à sa tangente.

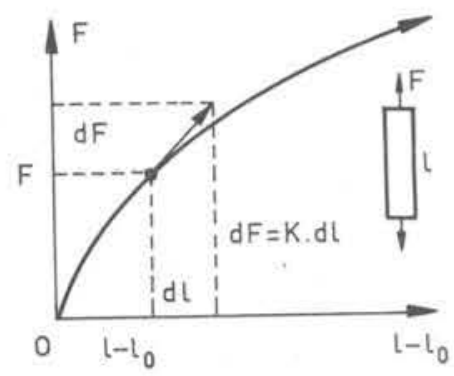

Fig. 1. - Schéma monodimensionnel en « charge ".

Notons que cette hypothèse n'implique un caractère ni linéaire ni réversible pour la loi.

Les expérimentateurs ont tous noté qu'un échantillon ayant suivi un certain chemin de sollicitation n'est plus isotrope. Nous avons tenu à nous affranchir de cette hypothèse d'isotropie en faisant celle beaucoup moins restrictive d'orthotropie, nous donnant ainsi la possibilité de prendre en compte une anisotropie généralement considérée comme suffisante pour un sol. La loi rhéologique incrémentale a donc été supposée orthotrope (c'est-à-dire symétrique par rapport à trois plans tri-orthogonaux).

Une autre caractéristique bien connue du comportement d'un sable est son irréversibilité, même aux faibles déformations. Cela se traduit par une courbe de " décharge " distincte de la " charge ".

Sur le schéma monodimensionnel (fig. 2), il nous faudrait ainsi distinguer deux valeurs de la fonction

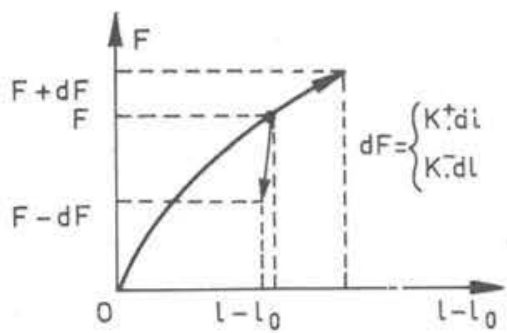

Fig. 2. - Schéma monodimensionnel en « charge » et en " décharge ".
$\mathrm{K}$ au point (F, $\left.l-l_{0}\right)$ : l'une correspondant à la " charge " et l'autre à la « décharge » :

$$
\begin{aligned}
& d l=1 / \mathrm{K}^{+} d \mathrm{~F}, d \mathrm{~F} \geqslant 0 \\
& d l=1 / \mathrm{K}^{-} d \mathrm{~F}, d \mathrm{~F} \leqslant 0
\end{aligned}
$$

Au niveau de la loi rhéologique, nous nous trouvons en présence de huit valeurs différentes pour la matrice $\mathrm{M}^{-1}\left(d \varepsilon=\mathrm{M}^{-1} d \sigma\right)$ suivant le signe de l'incrément principal de contrainte dans chaque direction d'orthotropie. Ces huit valeurs sont affectées à huit huitièmes d'espace (appelés zones tensorielles) dans l'espace des contraintes incrémentales principales. Remarquons qu'il devient alors abusif de parler en général de " charge " et de " décharge " puisque nous sommes en présence de huit zones (et non d'une zone de charge et d'une zone de décharge). On peut, par contre, utiliser les termes d' " aller " et de " retour ", en leur associant une orientation (un sens) sur un chemin de sollicitation donné.

Notre loi permet ainsi de décrire correctement les irréversibilités d'un milieu pulvérulent.

\subsection{Explicitation de la loi}

Il nous faut maintenant déterminer la matrice $\mathrm{M}^{-1}$, c'est-à-dire les douze termes non nuls qu'implique l'hypothèse d'orthotropie.

Supposons que le matériau ait atteint un état de contrainte représenté par le point $\mathrm{M}\left(\sigma_{1}, \sigma_{2}, \sigma_{3}\right)$. A la suite d'une petite sollicitation telle que les directions principales de contrainte restent fixes, l'état du milieu sera représenté par le point $\mathrm{P}\left(\sigma_{1}+d \sigma_{1}, \sigma_{2}+d \sigma_{2}\right.$, $\sigma_{3}+d \sigma_{3}$ ) (fig. 3). D'après l'hypothèse de linéarité (locale) de la loi incrémentale et en raisonnant dans la même zone tensorielle qui contient le chemin élémentaire réel MP, nous pouvons décomposer ce dernier en trois chemins élémentaires particuliers : $\mathrm{MA}, \mathrm{AB}, \mathrm{BP}$, pour lesquels une seule contrainte principale varie, les deux autres restant constantes mais distinctes (éventuellement).

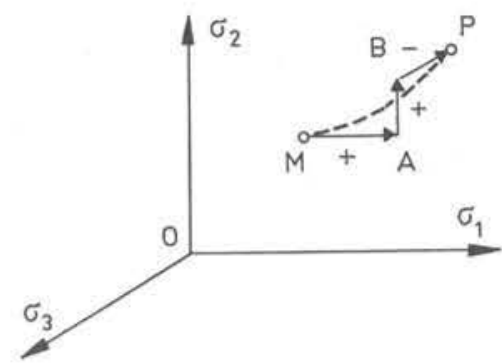

Fig. 3. - Décomposition d'un chemin de sollicitation élémentaire.

Nous avons alors montré (Darve [9]) que la loi rhéologique prenait la forme suivante :

$$
\left[\begin{array}{l}
d \varepsilon_{11} \\
d \varepsilon_{22} \\
d \varepsilon_{33} \\
d \varepsilon_{23} \\
d \varepsilon_{31} \\
d \varepsilon_{12}
\end{array}\right]=\left[\begin{array}{ccc}
1 / \mathrm{U}_{1} & -\mathrm{V}_{2}{ }^{1} / \mathrm{U}_{2} & -\mathrm{V}_{3}{ }^{1} / \mathrm{U}_{3} \\
-\mathrm{V}_{1}{ }^{2} / \mathrm{U}_{1} & 1 / \mathrm{U}_{2} & -\mathrm{V}_{3}{ }^{2} / \mathrm{U}_{3} \\
-\mathrm{V}_{1}{ }^{3} / \mathrm{U}_{1} & -\mathrm{V}_{2}{ }^{3} / \mathrm{U}_{2} & 1 / \mathrm{U}_{3} \\
\mathrm{O} & \mathrm{O} & \mathrm{O} \\
\mathrm{O} & \mathrm{O} & \mathrm{O} \\
\mathrm{O} & \mathrm{O} & \mathrm{O}
\end{array}\right.
$$$$
\begin{array}{cc}
\mathrm{O} & \mathrm{O} \\
\mathrm{O} & \mathrm{O} \\
\mathrm{O} & \mathrm{O} \\
2 \mathrm{G}_{1} & \mathrm{O} \\
\mathrm{O} & 2 \mathrm{G}_{2} \\
\mathrm{O} & \mathrm{O}
\end{array}
$$$$
\left.\begin{array}{l}
\mathrm{O} \\
\mathrm{O} \\
\mathrm{O} \\
\mathrm{O} \\
\mathrm{O} \\
\mathrm{G}_{3}
\end{array}\right]\left[\begin{array}{l}
d \sigma_{11} \\
d \sigma_{22} \\
d \sigma_{33} \\
d \sigma_{23} \\
d \sigma_{31} \\
d \sigma_{12}
\end{array}\right]
$$ 


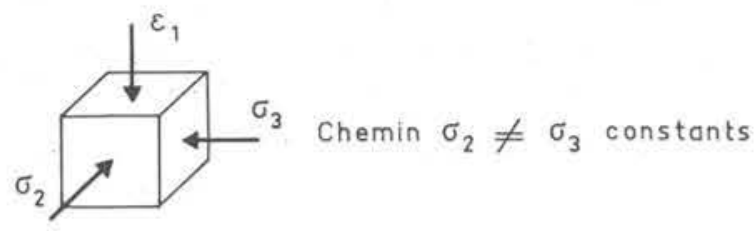

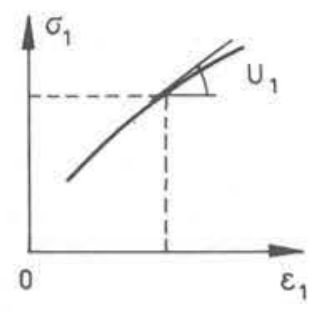

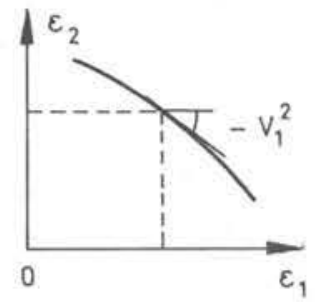

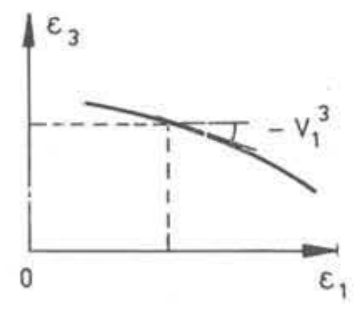

Fig. 4. - Représentation schématique des éléments de la matrice de la loi incrémentale.
Fig. 5, - Courbes expérimentales (sur chemin triaxial classique) nécessaires à la détermination des parametres de la loi.

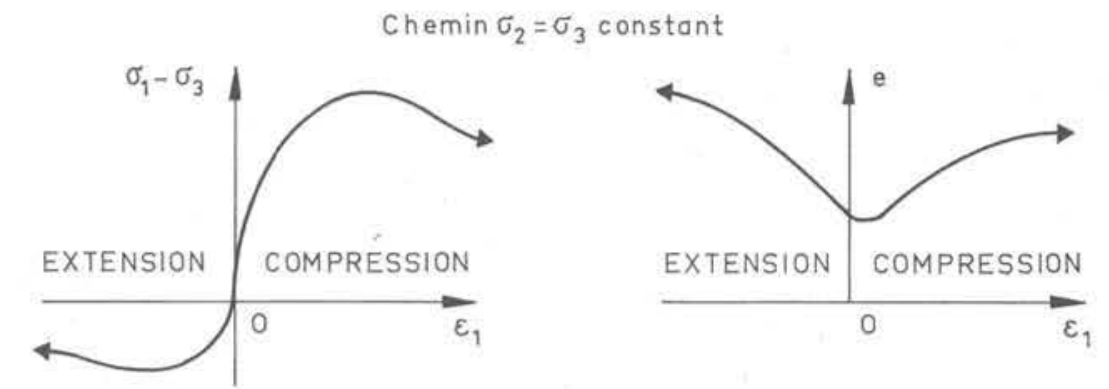

Les trois coefficients $\mathrm{U}_{k}$ et les six coefficients $\mathrm{V}_{k}{ }^{l}$ représentent les pentes de trois courbes effort-déformation et de six courbes de variations des déformations latérales, au point actuel en contraintes et en déformations, pour des chemins le long desquels une seule contrainte principale varie, les deux autres restant fixes. Ils sont fonction des états actuels de contrainte et de déformation et de la direction du vecteur sollicitation incrémentale actuel. Sur la figure 4 , nous avons schématisé la définition des éléments de la première colonne de la matrice de la loi, les courbes étant tracées pour des chemins pour lesquels $\sigma_{2}$ et $\sigma_{3}$ sont constants et différents.

.Les coefficients $G_{1}, G_{2}, G_{3}$ sont déterminés en fonction des précédents en supposant une variation sensiblement elfiptique de certaines " propriétés mécaniques » du milieu.

D'où par exemple :

$$
G_{1}=\frac{\sqrt{\left(U_{2}\right)^{2}+\left(U_{3}\right)^{2}}}{U_{2} U_{3} \sqrt{2}}+\frac{U_{2}\left(V_{3}{ }^{2}-1\right)+U_{3}\left(V_{2}{ }^{3}-1\right)}{4 U_{2} U_{3}}
$$

et $G_{2}$ et $G_{3}$ par permutation sur les indices.

Notons que la matrice n'est pas, a priori, symétrique.

\subsection{Détermination des paramètres de la loi d'un matériau donné}

Nous venons de voir que la connaissance d'une famille de courbes effort-déformation et de deux familles de courbes de variations des déformations latérales, ces courbes étant définies le long de chemins particuliers, suffirait pour décrire l'ensemble du comportement du matóriau.

En première approximation, nous avons formulé (Darve [9]) une expression analytique pour ces trois familles de courbes à partir de la donnée d'essais triaxiaux classiques, réalisés en compression et en extension. Un minimum de six essais pour trois valeurs différentes de la contrainte latérale suffit pour le calcul des coefficients entrant dans ces formulations.

Les expériences doivent ainsi fournir pour trois valeurs de $\sigma_{3}$ les courbes schématisées sur la figure 5 .

Sur ce plan, notre objectif a été de décrire toutes les caractéristiques classiquement admises du comportement mécanique d'un sable sur chemin triaxial. Ainsi, module d'Young et coefficient de Poisson à l'origine dépendent de la pression moyenne $\sigma_{m}$ :

$$
\mathrm{E}=\mathrm{K}\left(\sigma_{\mathrm{m}}\right)^{n} \quad \nu=\frac{\mathrm{A}}{\mathrm{B}+\sigma_{\mathrm{m}}}
$$

L'angle de frottement en " pic » (quand il existe) est fonction de $\sigma_{3}$ (et plus généralement de la contrainte intermédiaire) :

$$
\operatorname{tg} \Phi_{\text {vic }}=\frac{A_{4}}{A_{5}+\sigma_{3}}+\operatorname{tg} \Phi
$$

comme la déformation correspondante :

$$
\left(\varepsilon_{1}\right)_{\text {pic }}=A_{1}-A_{2} \exp \left(-A_{3} \sigma_{3}\right)
$$

Le diagramme critique du matériau est pris en compte :

$$
e_{c}=\mathrm{B}_{1}+\mathrm{B}_{2} \exp \left(-\mathrm{B}_{3} \sigma_{m}\right)
$$

$\sigma_{m}$ étant la pression moyenne en "palier » de plasticité.

Le minimum de l'indice des vides (quand il existe) est lui aussi caractérisé.

La définition des coefficients $\mathrm{K}, n, \mathrm{~A}, \mathrm{~B}, \ldots$ suppose qu'il a été défini un état de référence, isotrope en contraintes, du matériau, sa densité (initiale) étant donnée pour cet état.

Sur la figure 6 , nous montrons un exemple de chemin triaxial classique $\left(\sigma_{3}=0.6 \mathrm{daN} / \mathrm{cm}^{2}\right)$ sur sable dense (points pleins) et sable lâche (points évidés), ayant 


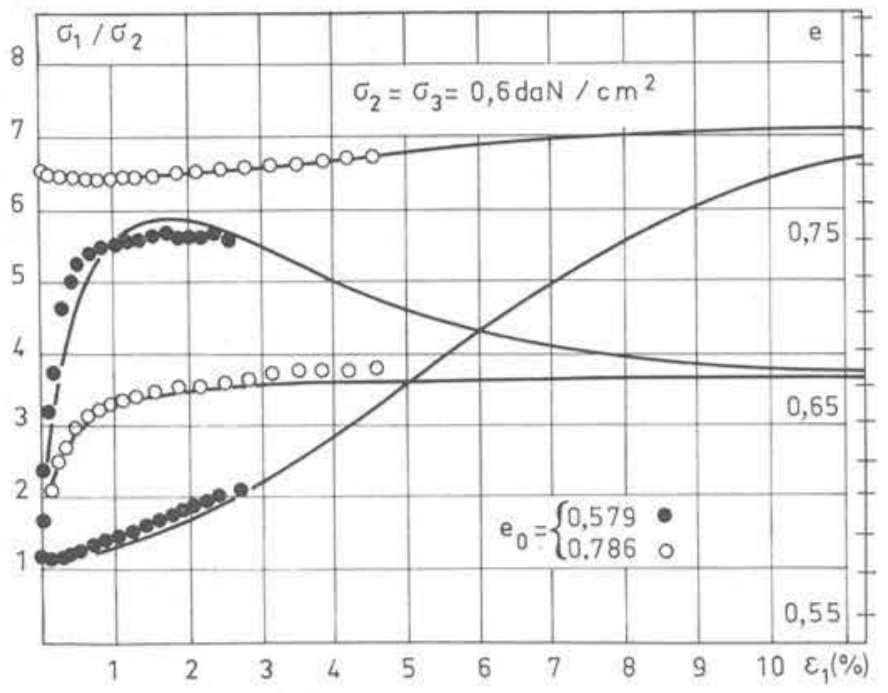

permis le calcul par Labanieh [10] des paramètres de la loi du sable considéré à forte et faible densités. Les points représentent des mesures expérimentales effectuées par Lade [11] et les courbes notre formulation analytique approchée.

Notons que le comportement d'un sable dense (fort maximum de contrainte et dilatance importante lors d'un écrasement au triaxial) comme celui d'un sable lâche (pas de « pic ", faibles variations de volume) sont correctement décrits.

Remarquons enfin que la formulation analytique utilisée et les formules empiriques données pourraient être avantageusement remplacées par une entrée numérique point par point sur ordinateur des valeurs expérimentales.

Fig. 6. - Exemple de chemin triaxial classique ayant permis le calcul des paramètres de la loi. Points expérimentaux et courbes analytiques. Cas d'un sable à forte et faible densités.

\section{APPLICATION DE LA LOI A DES CHEMINS DE SOLLICITATION HOMOGENES ET COMPARAISONS AVEC L'EXPERIENCE}

Les coefficients des sables dense et lâche ayant été calculés précédemment à partir des chemins triaxiaux, nous avons pu intégrer la loi (ainsi déterminée) sur différents autres chemins de sollicitation et comparer les résultats théoriques avec les mesures expérimentales effectuées par Lade et Duncan ([11] et [12]).

La figure 7 présente ainsi une comparaison points expérimentaux-courbes théoriques sur un chemin simple : isotrope.

L'appareil le plus complet existant à l'heure actuelle en mécanique des sols est l'appareil « vraiment " triaxial, ou presse tridimensionnelle, sur laquelle les trois déformations et les trois contraintes principales peuvent être différentes. Il est donc très intéressant de vérifier la validité d'une loi rhéologique sur un tel appareil, permettant de suivre une gamme très vaste de chemins de sollicitation.

\subsection{Chemins en contraintes planes}

Sur leur « vrai » triaxial, Lade et Duncan ont suivi des chemins en contraintes planes définies par :

direction 1 : écrasement

direction 2 : contrainte constante : $\sigma_{2}=0.6 \mathrm{daN} / \mathrm{cm}^{2}$ direction 3 : accroissement de contrainte asservi par : $\Delta \sigma_{3}=\mathrm{B} \Delta \sigma_{1} \quad$ avec B constant.

De l'ensemble des calculs faits, nous présentons trois exemples :

sable dense, $B=0.15$ et 0.50

sable lâche, $\mathrm{B}=1$.

Pour chacun de ces trois cas, nous présentons (fig. 8, $9,10)$ une courbe effort-déformation : $\sigma_{1}-\sigma_{2}=f\left(\varepsilon_{1}\right)$, les variations de l'indice des vides : $e$ et les deux courbes de variations des déformations latérales : $\varepsilon_{2}$ et $\varepsilon_{3}$.

La grandeur de l'incrément de sollicitation $\Delta \varepsilon_{1}$ choisi pour l'intégration numérique était égal à : $0.03 \%$. Toutes les courbes présentées ont été directement tracées par ordinateur.

Commentons rapidement les résultats.

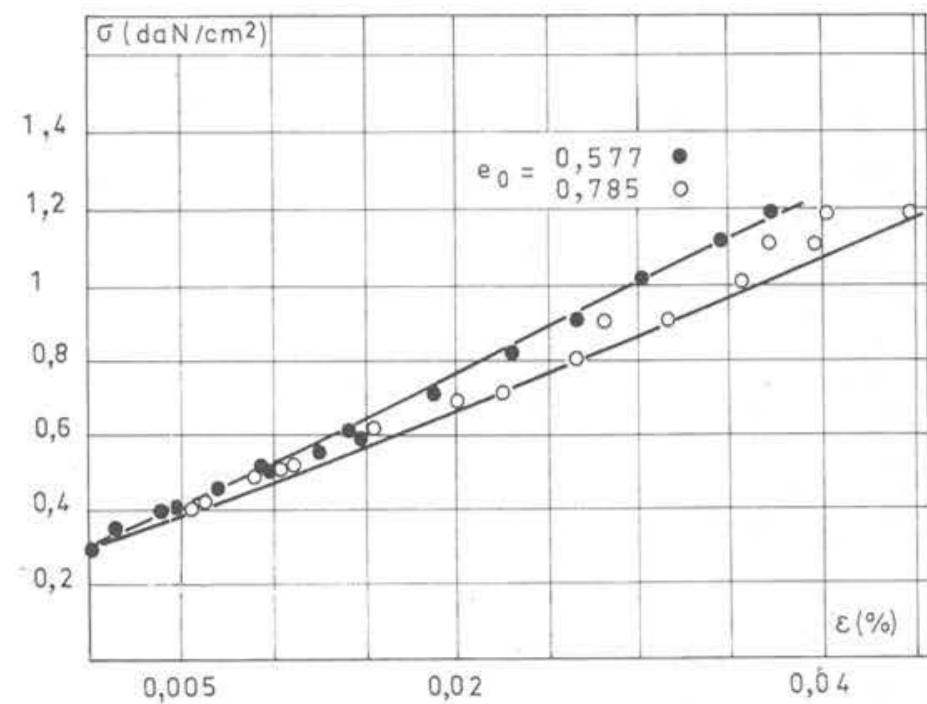

Fig. 7. - Chemins isotropes. Points expérimentaux et courbes théoriques résultant de l'intégration de la loi incrémentale.

Notons tout d'abord, dans le cas du sable dense, l'importance du " pic ", supérieur à ce que donnerait le critère de Coulomb. Notre critère de plasticité rend compte effectivement de cette influence de la contrainte intermédiaire.

Par ailleurs, au fur et à mesure que B augmente, la contrainte $\sigma_{3}$ croît et la déformation correspondante $\varepsilon_{3}$ passe de valeurs négatives $(B=0.15)$, à des valeurs faiblement positives $(B=0.50)$, puis nettement positives $\left(B=1\right.$ et $\left.\varepsilon_{3}=\varepsilon_{1}\right)$.

\subsection{Chemins en déformations planes}

Les autres chemins suivis par Lade et Duncan sur leur "vrai " triaxial sont constitués par les chemins en déformation plane, définis ci-après :

direction 1 : écrasement 


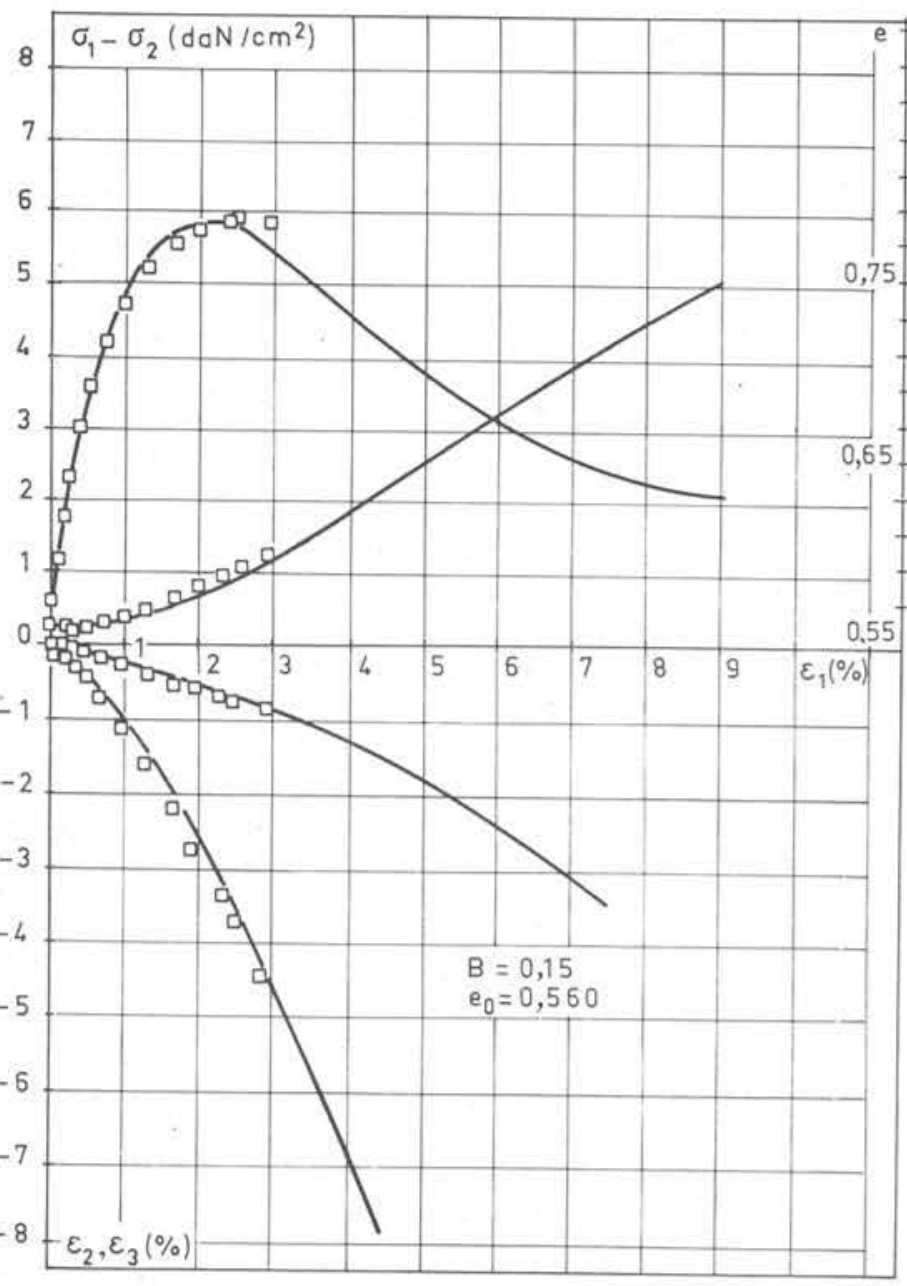

Fig. 8. - Chemin en contraintes planes avec $\mathbf{B}=\mathbf{0 , 1 5}$ sur le sable dense. Comparaison théorie-expérience.

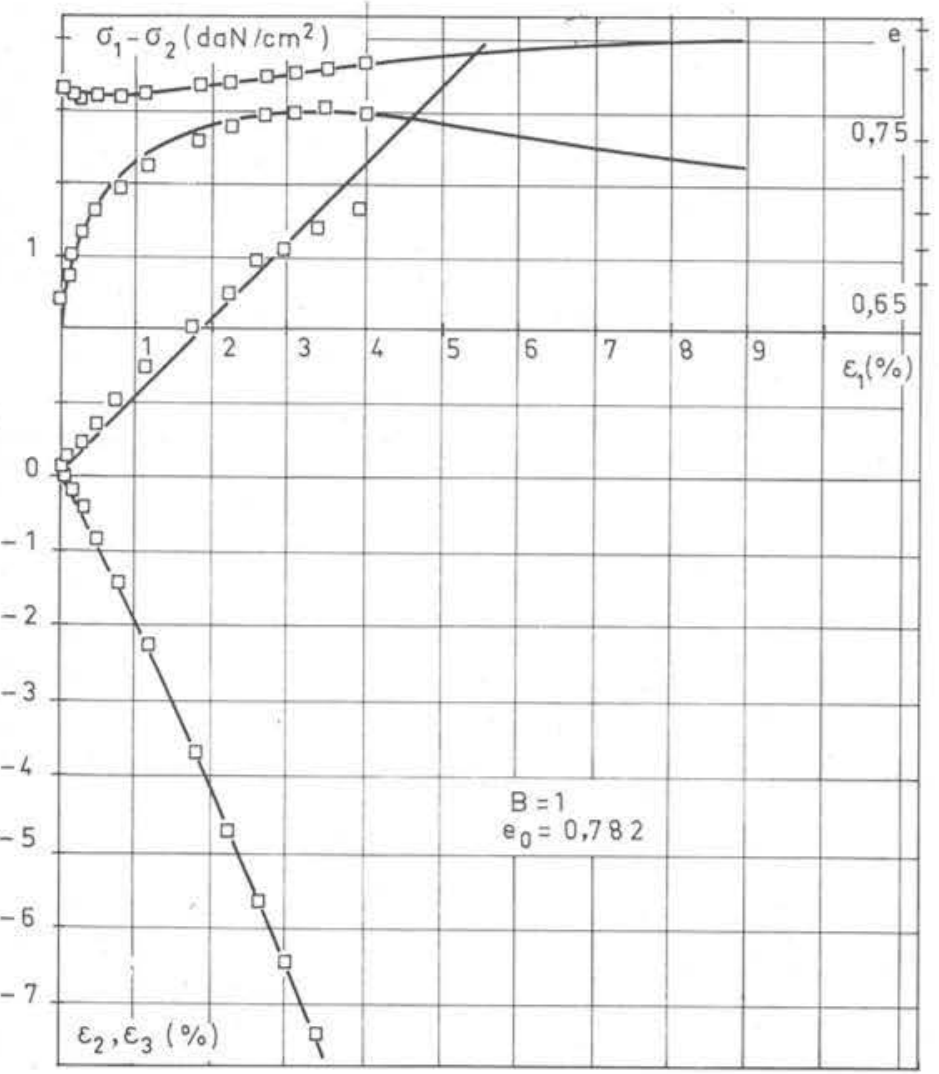

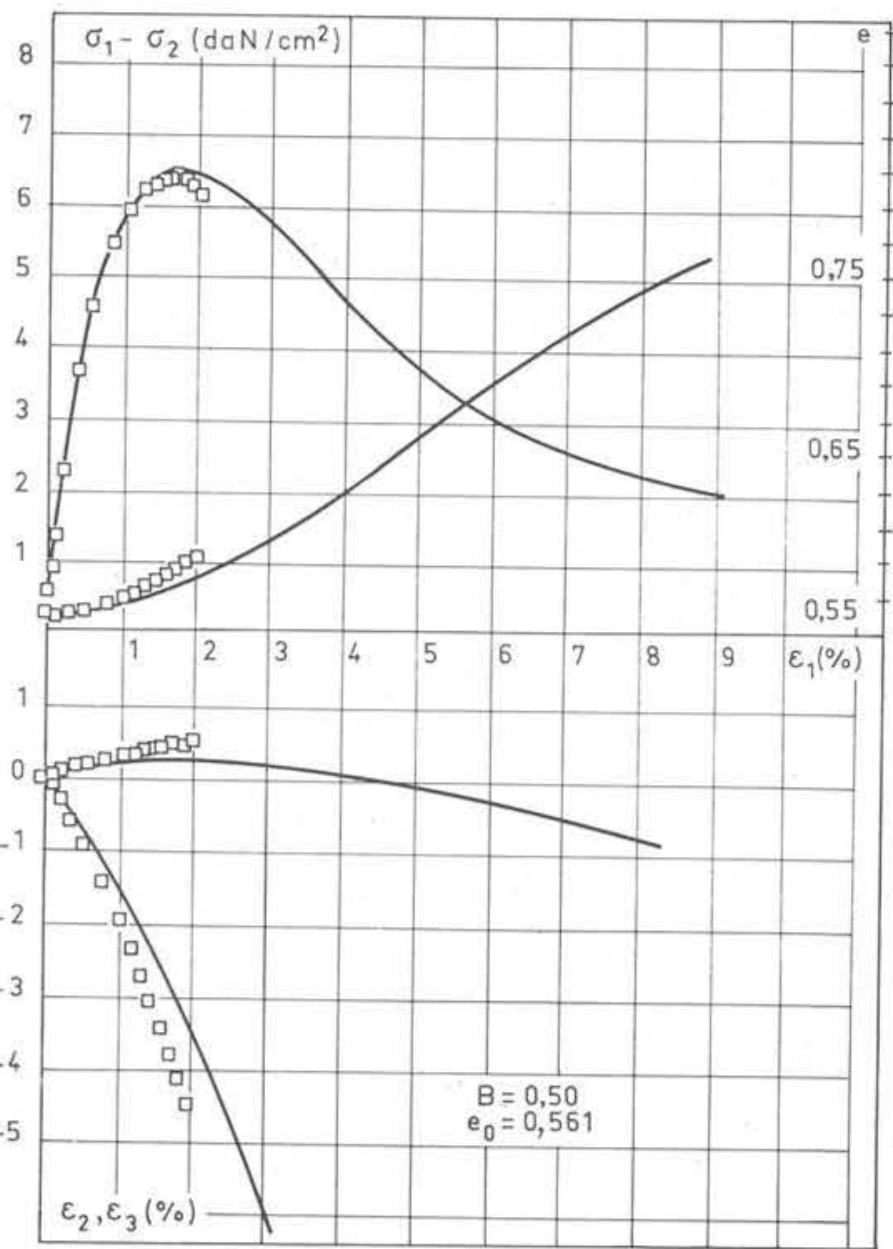

Fig. 9. - Chemin en contraintes planes avec $\mathbf{B}=\mathbf{0 , 5 0}$ sur le sable dense.

direction 2 : contrainte constante : $\sigma_{2}=0.6 \mathrm{daN} / \mathrm{cm}^{2}$ direction 3 : déformation plane : $\varepsilon_{3}=0$.

Nous présentons, sur la figure 11 , les points expérimentaux comparés aux courbes théoriques dans les deux cas du sable dense et lâche. Nous avons étudié les deux courbes effort-déformation (dans les directions 1 et 3 ), une courbe de variation des déformations latérales dans la direction 2 de contrainte plane et les variations de l'indice des vides $e$.

Remarquons la disparition du « pic » pour la courbe effort-déformation relative à la direction 3 de défor mation plane. L'angle de frottement de pic que l'on définit de manière classique en déformation plane (c'est-à-dire celui relatif à la direction 1 d'écrasement), déduit de la courbe théorique, est en bonne concor dance avec l'expérience. Les valeurs des déformations sont aussi satisfaisantes.

Fig. 10. - Chemin en contraintes planes avec $B=10$ sur le sable lâche. 

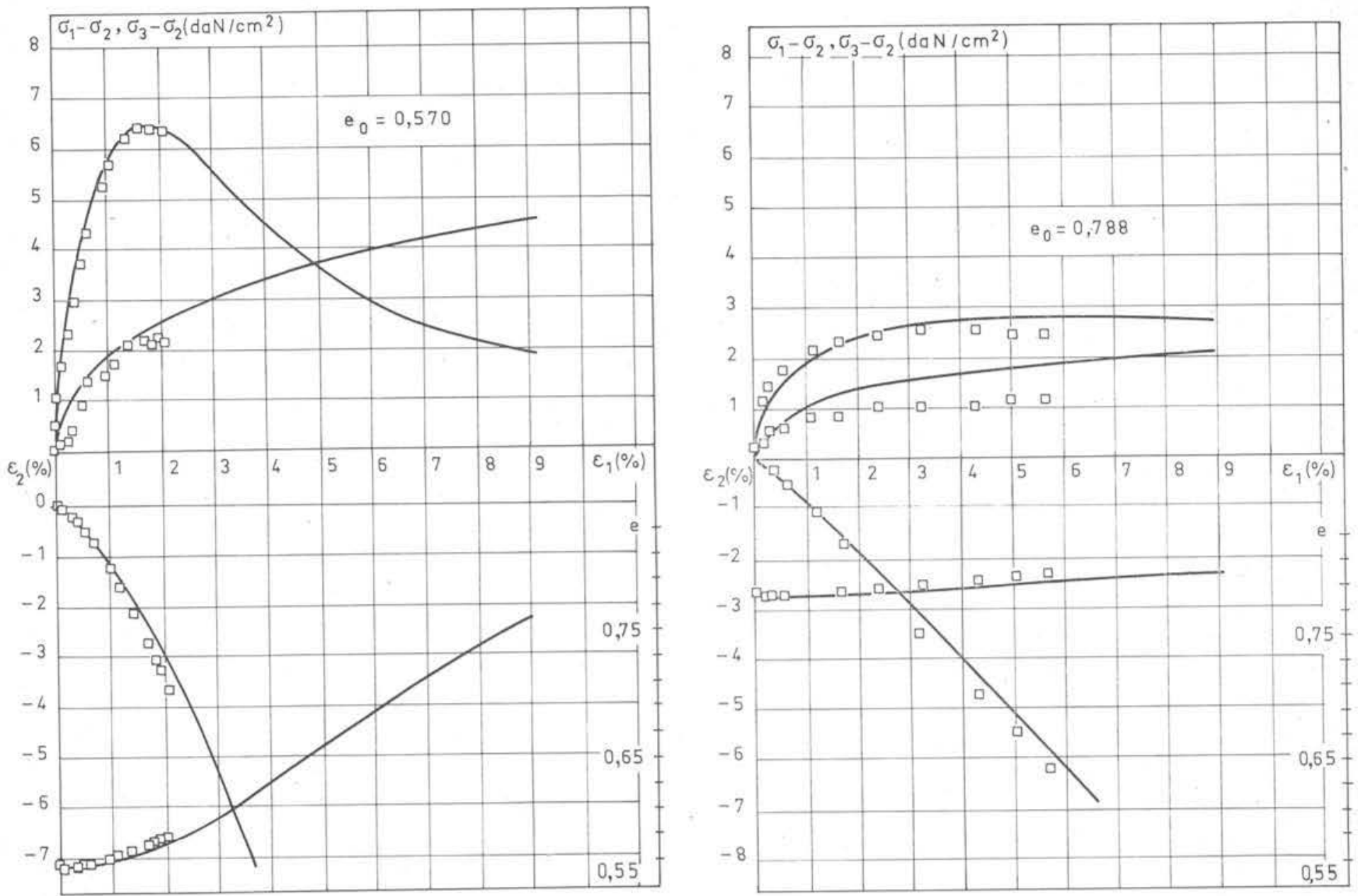

Fig. 11. - Chemin en déformations planes dans la direction 3 et en contraintes planes dans la direction 2 dans le cas du sable à forte et faible densités.

\section{QUELQUES CALCULS AUX LIMITES UTILISANT LA LOI INCREMENTALE EFFECTUES PAR LA METHODE DES ELEMENTS FINIS}

\subsection{Introduction et hypothèses}

Nous présentons ci-dessous des résultats obtenus en utilisant la loi incrémentale que nous venons de décrire. Pour résoudre ces problèmes aux limites nous utilisons la méthode des éléments finis. Nous n'en ferons pas une présentation détaillée, et nous renvoyons le lecteur aux ouvrages spécialisés (Zienkiewicz [2]). Cependant, la loi utilisée liant l'incrément de contrainte à l'incrément de déformation, et cette loi dépendant du chemin de sollicitation suivi antérieurement pour chaque point, il est nécessaire d'effectuer le calcul pas à pas, c'est-à-dire en imposant des incréments de conditions aux limites.

Les calculs que nous présentons sont des simulations numériques de modèles de laboratoire constitués de petits rouleaux (matériau de Schneebeli [13]). Les problèmes sont donc naturellement plans. Les expériences correspondantes ont été réalisées par Pioline [14].

Avant de présenter les résultats de nos calculs nous voudrions dégager les hypothèses simplificatrices que nous avons utilisées et qui en limitent la validité. Ces hypothèses sont de deux natures différentes. En premier lieu, nous examinerons les hypothèses relatives à la loi, ensuite nous exposerons celles qui portent sur les problèmes traités.

a) Hypothèses simplificatrices faites sur la loi

Nous avons supposé que la matrice $\mathrm{M}$, représentatrice de la loi rhéologique incrémentale, était symétrique,

Au niveau des données, les courbes effort-déformation sur chemins triaxiaux classiques, nécessaires à la détermination des paramètres, sont simplifiées : on le suppose être sans " pic ». Cette approximation est relativement justifiée du fait que les pics sont relativement peu marqués pour le matériau de Schneebeli.

Enfin, nous avons fait l'hypothèse que les axes d'orthotropie de la loi incrémentale étaient confondus avec les directions principales de déformation totale (c'est-à-dire prise par rapport à l'état de référence).

b) Hypothèses simplificatrices relatives aux problèmes traités

Les problèmes que nous traitons seront supposés tels que, pour tout point du massif considéré, d'une 


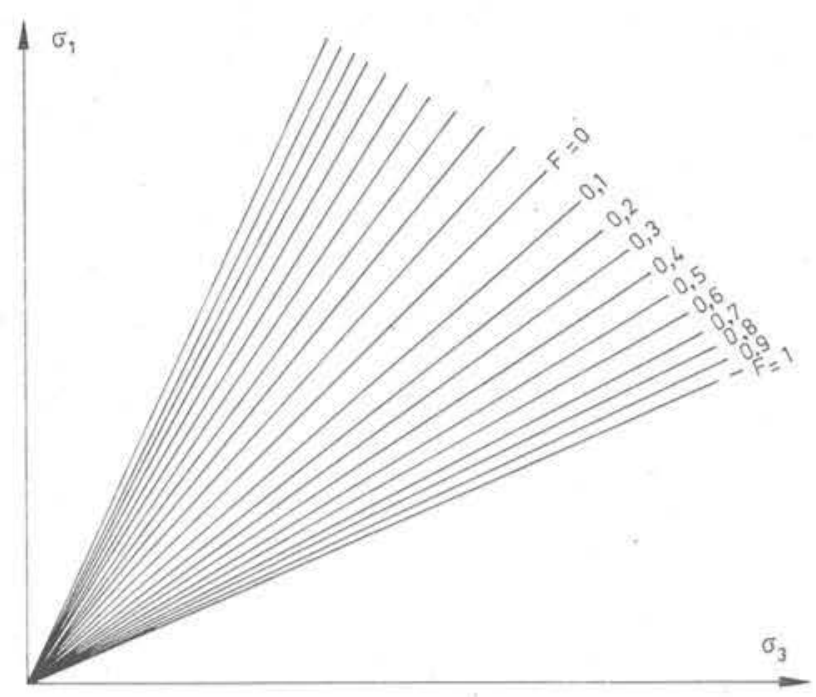

$$
F=\frac{\sigma_{1} / \sigma_{3}-1}{\operatorname{tg}^{2}\left(\frac{\pi}{4}+\frac{\Phi}{2}\right)-1}
$$

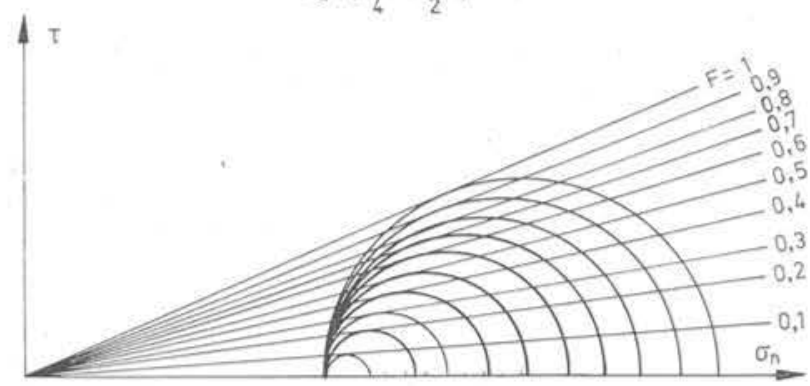

Fig. 12. - Interprétation du coefficient F.

part les déformations et les rotations totales sont supposées petites, d'autre part il n'y a pas de changement de sens de la sollicitation locale. (En d'autres termes au cours de l'évolution du problème, aucun point ne change de zone tensorielle.) A l'issue d'un calcul donné on peut vérifier que ces deux hypothèses sont vérifiées ou non. Elles restreignent bien entendu le champ des problèmes que l'on peut traiter actuellement.

- Enfin, rappelons que la loi est nécessairement intégrée à partir de l'état de référence du matériau. Il convient donc de faire une hypothèse relative au chemin suivi par tout point du massif depuis l'état de référence jusqu'à l'état initial du problème. Dans tous les problèmes que nous présentons nous avons supposé, pour chaque point du massif, que ce chemin d'initialisation était isotrope, ce qui a comme conséquence que le $\mathrm{K}_{o}$ initial est égal à 1 .

\subsection{Présentation des résultats}

Il nous a paru significatif de tracer l'évolution dans le temps de la variation dans l'espace du coefficient $F$.

$$
\mathrm{F}=\frac{\sigma_{1} / \sigma_{3}-1}{\operatorname{tg}^{2}(\pi / 4+\Phi / 2)-1}
$$

$\sigma_{1}$ est la contrainte principale majeure, $\sigma_{3}$ la contrainte principale mineure, $\Phi$ l'angle de frottement interne de palier $\Phi=22^{\circ} 5$. Pour cela nous avons tracé, pour des états successifs du massif de sol variant de l'état initial à l'état final du problème, les cartes d'isovaleurs de F.
Remarquons que $\mathrm{F}$ peut être interprété comme l'inverse d'un coefficient de sécurité local et que, à cause de l'initialisation, au début de tous nos problèmes $F=0$ en tout point. D'autre part si le palier de plasticité est atteint, $\mathrm{F}=1$; la croissance de $\mathrm{F}$ nous donnera la tendance à la plasticité.

Sur la figure 12 nous avons tracé, d'une part dans le plan des contraintes principales, d'autre part dans le plan de Mohr, les courbes engendrées par les points ayant des valeurs données de F.

\subsection{La simulation d'un essai triaxial parfaitement fretté en tête et en pied}

Nous avons simulé un essai triaxial fretté de $20 \mathrm{~cm}$ de haut sur $10 \mathrm{~cm}$ de large. On a négligé la pesanteur. En conséquence, nous n'avons étudié que le quart de l'échantillon, divisé en cinquante éléments (soixante six noeuds) égaux. Tous les points sont supposés avoir un même état initial isotrope à 0.1 bar. On a supposé un frottement parfait entre la tête (et le pied) de l'appareil et l'échantillon.

Le calcul a été fait en quinze incréments égaux correspondant chacun à une déformation moyenne de $1 \times 10^{-3}$.

La figure 13 représente la courbe contrainte (moyenne)-déformation (totale, c'est-à-dire calculée à partir du déplacement de la tête), et les variations d'indice des vides en tête (moyenne sur les cinq points) et au centre de l'échantillon (moyenne).

On retrouve sur la figure 14 l'évolution de la distribution de l'indice des vides dans l'échantillon, au cours du temps. Signalons qu'il manque sur la figure 14 les isovaleurs 0.230 et 0.231 .

La figure 15 nous permet de voir l'apparition de la "zone plastique». On peut remarquer que les zones proches de la tête de l'échantillon sont loin de la plasticité.
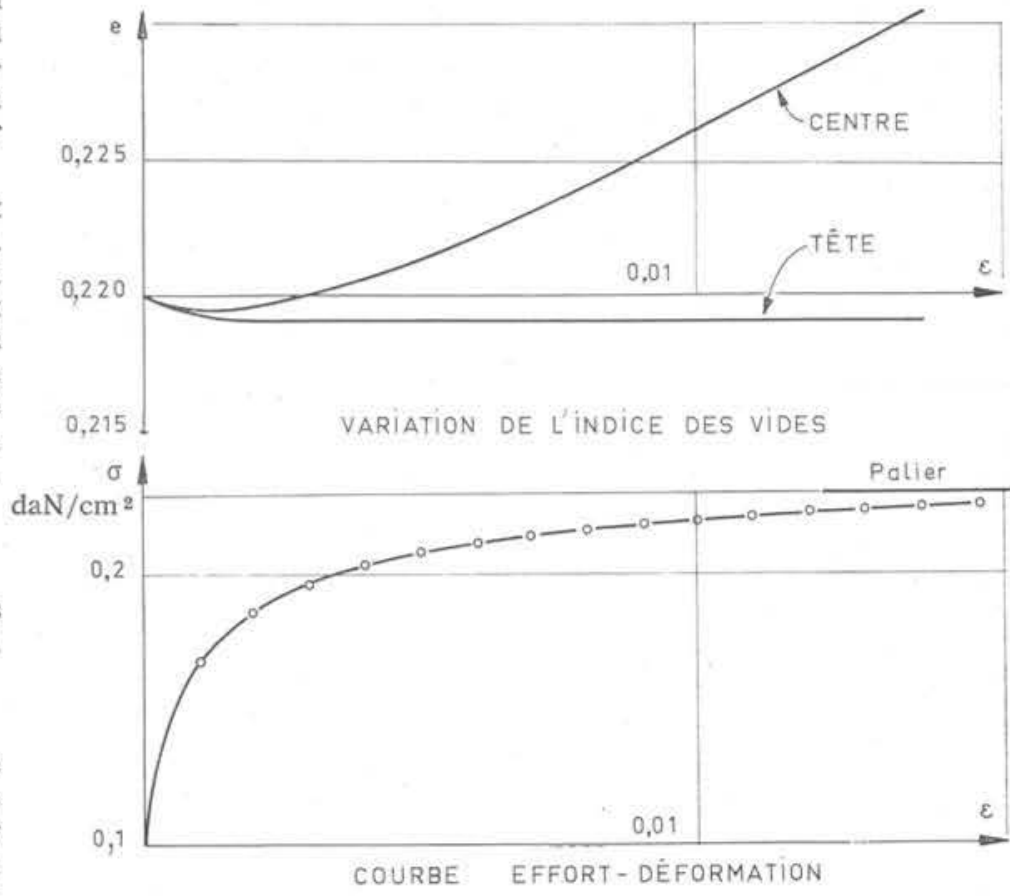

Fig, 13. - Essai triaxial fretté. 

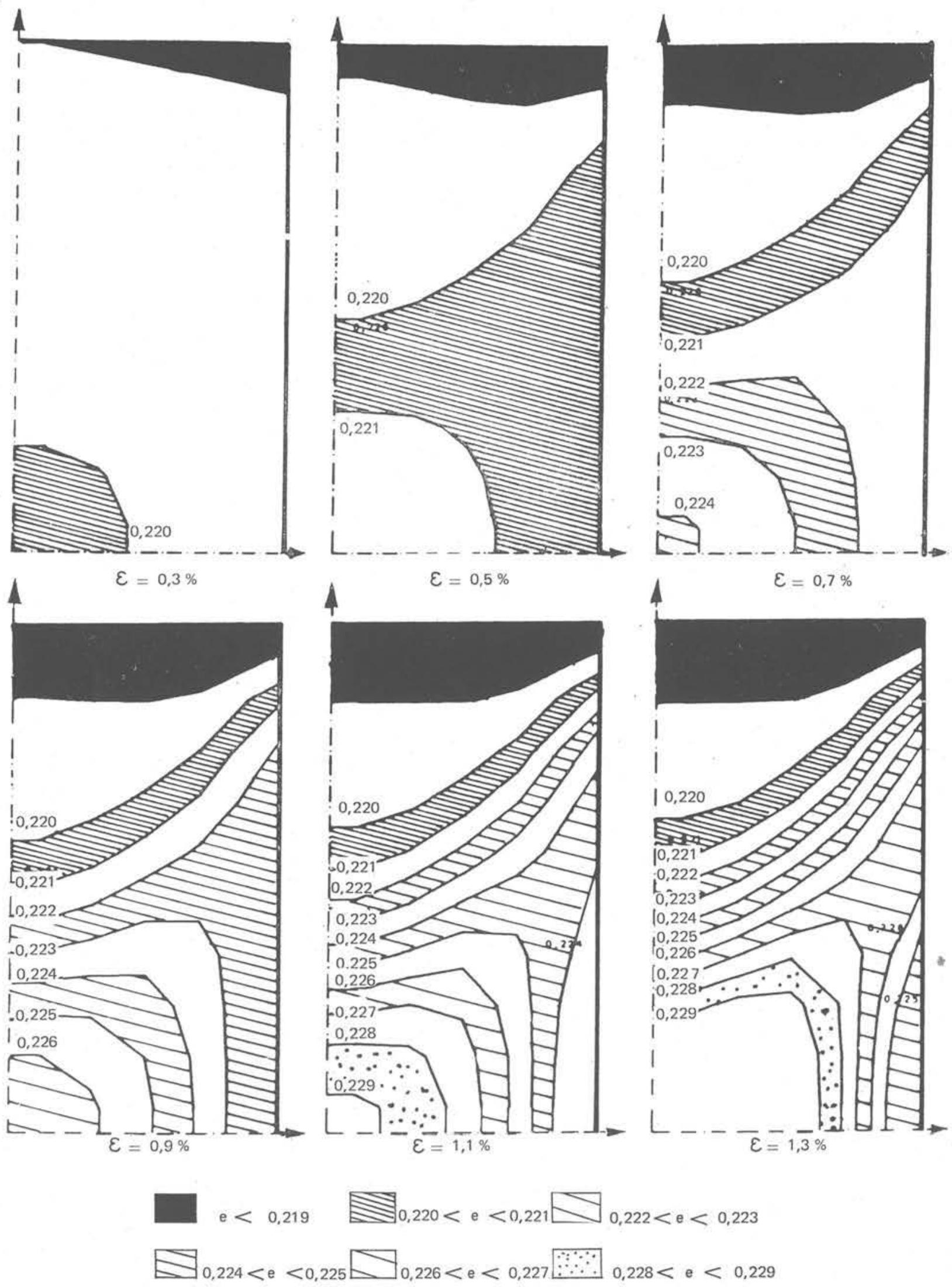

Fig. 14. - Triaxial fretté, évolution de l'indice des vides. 


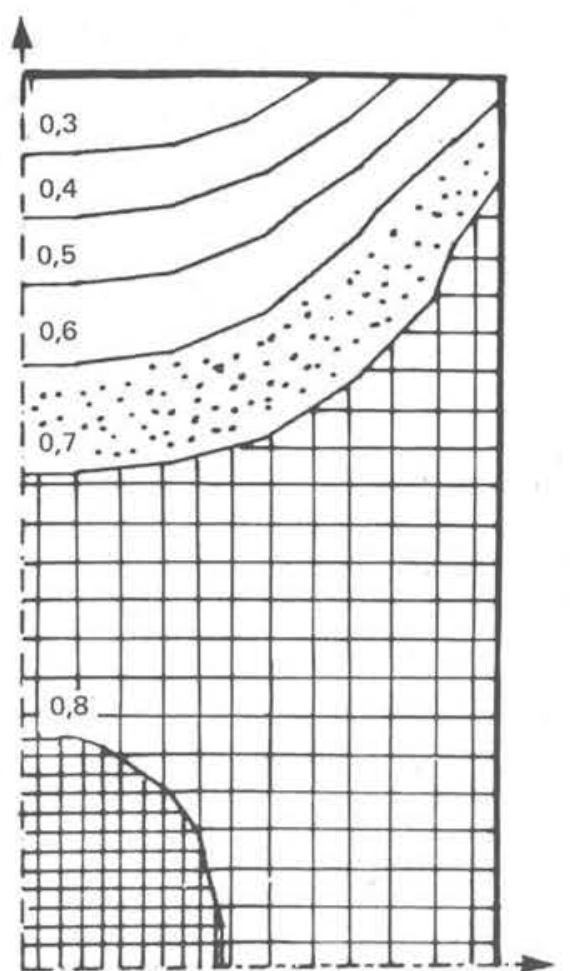

$\varepsilon=0,3 \%$

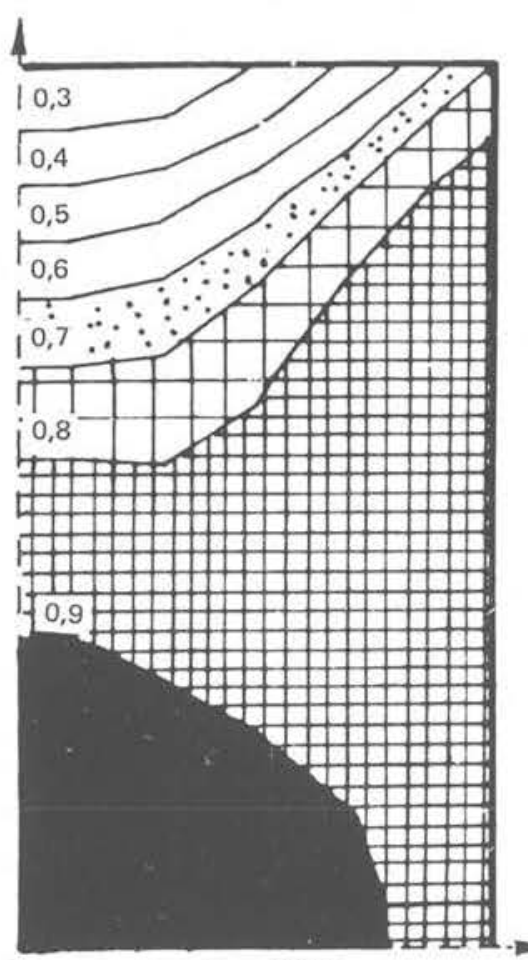

$\varepsilon=0,9 \%$
4
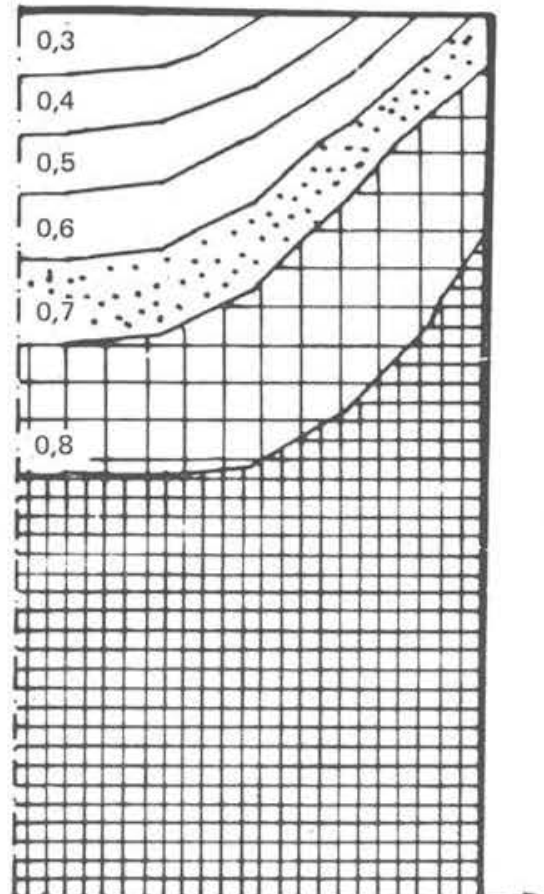

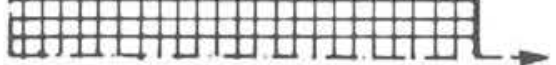

$\varepsilon=0,5 \%$

1

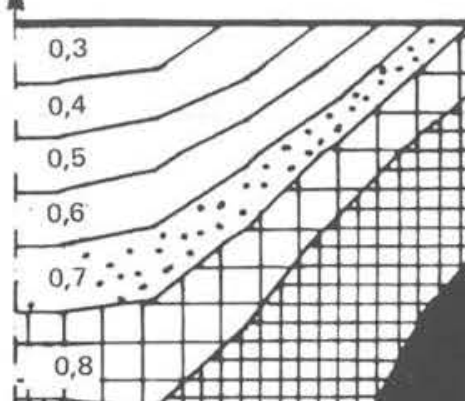

II,

\#An

且 40

H $10+24$

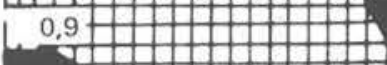

थम 1 Pी

भम+201

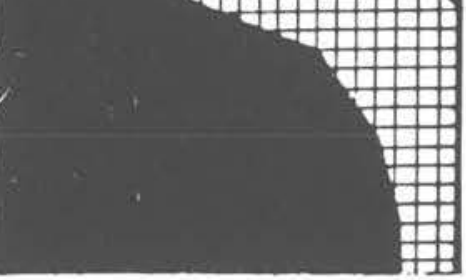

$\mathcal{E}=1,1 \%$
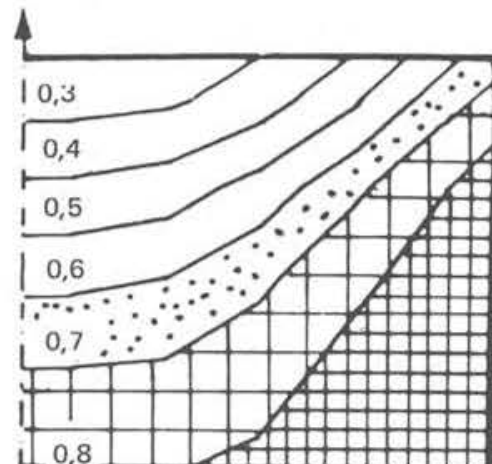

0,8 乎种共

计圳

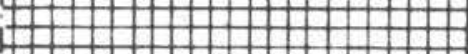

In 2 A

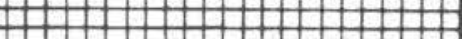

H1 2 H

$-0,9 \mathrm{Y}+\mathrm{W}$

mand

\begin{tabular}{l} 
म \\
\hline
\end{tabular}

भ

4 H भ19HA भ+1

$\varepsilon=0,7 \%$
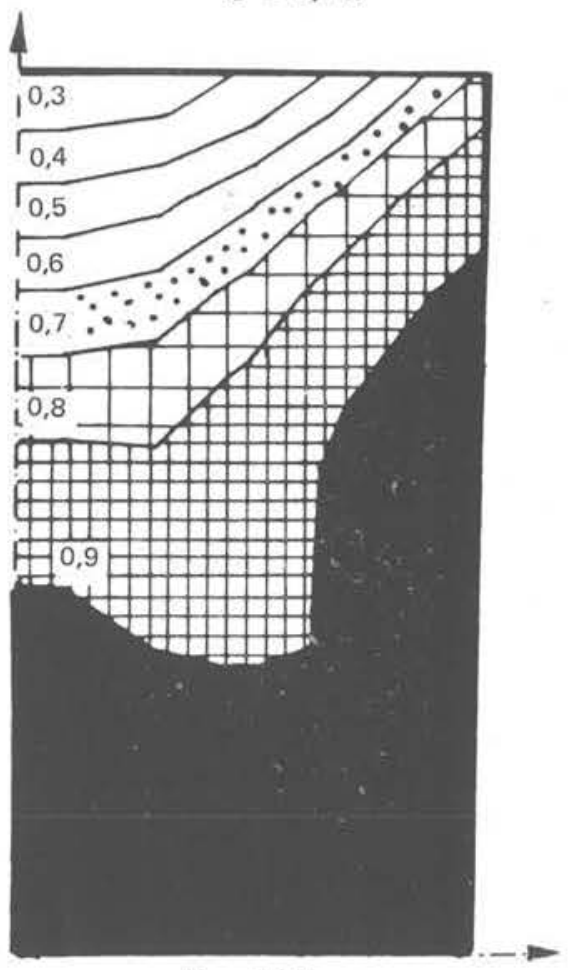

$\mathcal{E}=1,3 \%$

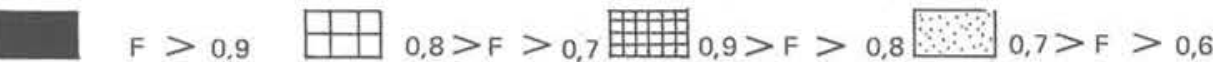

Fig. 15. - Triaxial fretté, évolution de $\mathbf{F}=\frac{\sigma_{1} / \sigma_{3}-1}{\operatorname{tg}^{2}(\pi / 4+\Phi / 2)}$ 
Malheureusement, il n'y a pas eu d'expérience correspondant à nos calculs (il $\mathrm{y}$ a de gros problèmes expérimentaux, tels que la mise en place, pour obtenir une bonne homogénéité initiale et des mesures fines de l'indice des vides). Soulignons, néanmoins que l'on retrouve qualitativement les résultats obtenus par Wack [15] et Terriez [16] sur des triaxiaux de sables, qui illustrent comme le calcul la très grande hétérogénéité des échantillons.

\subsection{Enfoncement d'un modèle de fondation rigide et rugueux}

\subsection{Définition et position du problème}

Nous avons effectué un calcul simulant l'enfoncement d'une plaque, rigide et rugueuse, dans un massif de petits rouleaux (matériau de Schneebeli). Grâce à la symétrie du problème, le calcul ne porte que sur la moitié du massif. La géométrie et le maillage sont représentés sur la figure 16 . Le massif a $80 \mathrm{~cm}$ de profondeur sur $1 \mathrm{~m}$ de demi-largeur. L'essai est effectué avec une plaque de $10 \mathrm{~cm}$ de demi-largeur.

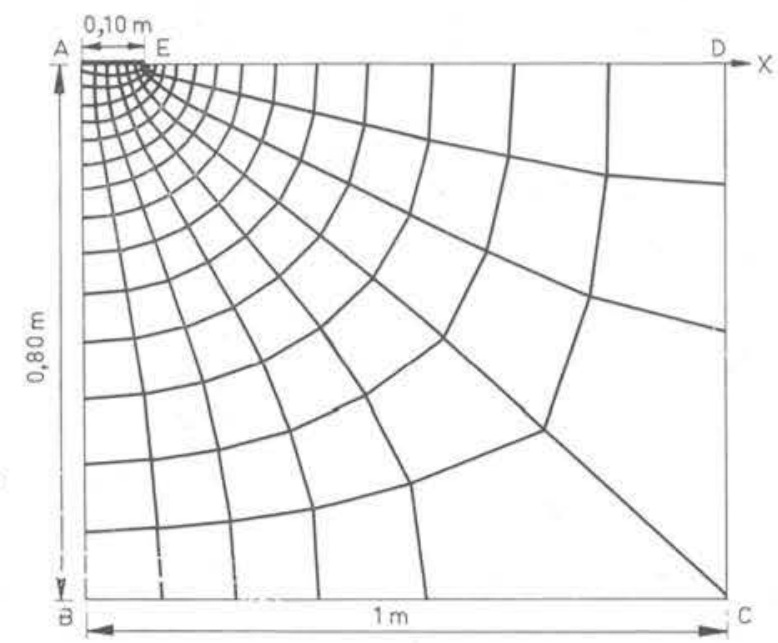

Fig. 16. - Enfoncement de plaque de $20 \mathrm{~cm}$ : maillage et géométrie.

En conséquence, les conditions aux limites sont les suivantes : sur $\mathrm{AB}$, l'axe de symétrie, déplacement horizontal nul, contrainte tangentielle nulle; sur $B C$ et $C D$ déplacements nuls; sur DE pression nulle; enfin sur $\mathrm{AB}$ (la demi-plaque), le déplacement horizontal est supposé nul et tous les déplacements verticaux égaux (à w).

Le maillage, qui comporte cent vingt éléments quadrilatéraux (cent quarante-quatre noeuds), est réalisé par un programme de maillage automatique basé sur la théorie des transformations conformes, décrit dans Chambon [17], qui permet une description plus fine au voisinage de la plaque.

Chaque point du massif est initialisé à l'état isotrope correspondant à sa contrainte "hydrostatique ".

Le problème a été résolu en dix incréments d'enfoncement variant en progression arithmétique, de façon qu'en aucun point, l'état de contrainte ne sorte de la surface limite. L'idéal serait de moduler l'incrément en fonction de la variation des termes de la matrice de la loi incrémentale. L'enfoncement total a été de $3 \mathrm{~mm}$.

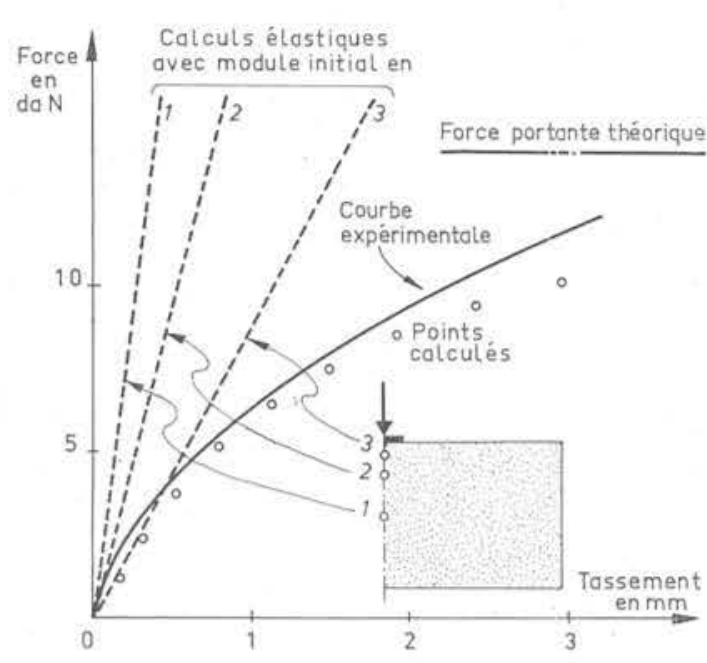

Fig. 17. - Enfoncement de plaque de $20 \mathrm{~cm}$ : comparaison des courbes « force-enfoncement " calculée et mesurée.

\subsection{Résultats}

La figure 17 présente les résultats qui nous semblent les plus importants : la comparaison de nos calculs avec les expériences de Pioline [6]. Nous avons placé sur cette figure, d'une part la force portante maximale théorique (plasticité parfaite) pour un enfoncement nul, correspondant à l'angle de palier $\Phi=22^{\circ} 5$ puisque notre calcul est fait sans pic, et d'autre part les résultats de calculs élastiques faits en prenant comme module d'Young la pente initiale des courbes triaxiales classiques pour trois pressions latérales. Ces pressions latérales étant égales aux contraintes verticales dues au poids des terres situées au-dessus des trois points 1,2 et 3 notés sur la figure.

La coïncidence entre le calcul et l'expérience se révèle être relativement bonne puisqu'au terme du calcul, il correspond environ aux deux tiers de la force portante théorique.

Cependant, les résultats des calculs sont systématiquement inférieurs aux mesures. Nous pensons qu'il y a deux raisons à cela : en premier lieu, le calcul néglige l'existence du «pic »; ensuite le calcul, considérant confondues toutes les géométries successives du massif, ne peut tenir compte du fait que l'enfoncement de la plaque fait intervenir un terme de surcharge (voir calcul classique des forces portantes de fondations, Terzaghi [18]). Si, par exemple, on calcule ce terme de surcharge pour un enfoncement de $3 \mathrm{~mm}$, on voit qu'il est égal à environ la moitié de la différence entre calcul et expérience. Ceci explique d'ailleurs que l'erreur grandisse avec le tassement.

La figure 18 représente l'évolution des contraintes normales sous la plaque. On peut remarquer que l'on retrouve pour les grands enfoncements, l'allure des courbes données par les mesures de Turpain [19] et les calculs de Stutz [20] en plasticité parfaite. D'autre part, notons que la contrainte n'est pas rigoureusement nulle au bord, bien que l'on ait pris soin de prendre les premiers incréments petits. On retrouve au milieu de l'évolution, une courbe avec un léger "creux " de la contrainte au centre de la plaque.

La figure 19 représente l'évolution de la répartition dans l'espace de F. On peut y voir l'entrée progressive du sol en plasticité au droit du bord de la fondation. 

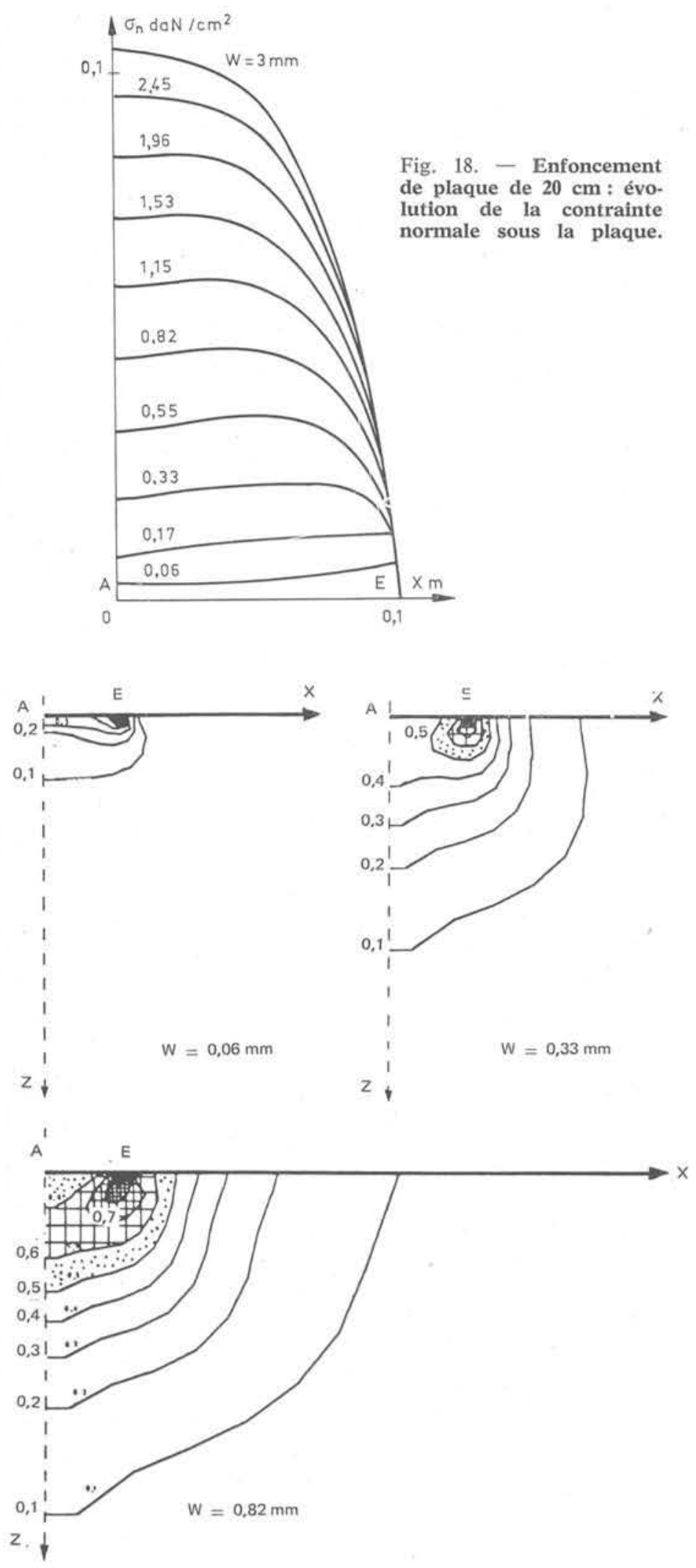

\subsection{Translation d'un écran vertical vers l'extérieur d'un massif}

\subsection{Définition et position du problème}

La géométrie et le maillage sont représentés sur la figure 20. Le massif mesure $92.5 \mathrm{~cm}$ de hauteur, et $135 \mathrm{~cm}$ de largeur; l'écran $A B$ fait $70 \mathrm{~cm}$ de hauteur et il est supposé parfaitement rugueux. Les conditions aux limites sont les suivantes : les déplacements sur $\mathrm{BDCE}$ sont nuls; la contrainte sur EA est nulle ; sur $A B$, les déplacements verticaux sont nuls et les déplacements horizontaux égaux (à $\Delta x$ ).

Le maillage qui comporte cent vingt éléments (cent quarante-trois noeuds) a été réalisé par le même programme que précédemment; le but recherché était d'avoir des mailles petites le long de l'écran.

L'initialisation est identique à celle des problèmes précédents.

La translation de l'écran $(2 \mathrm{~mm})$ est divisée en dix incréments variant en progression arithmétique pour les mêmes raisons que dans le cas des essais de plaque.

Avant d'examiner les résultats, il faut noter que les conditions de l'expérience correspondante ne sont pas
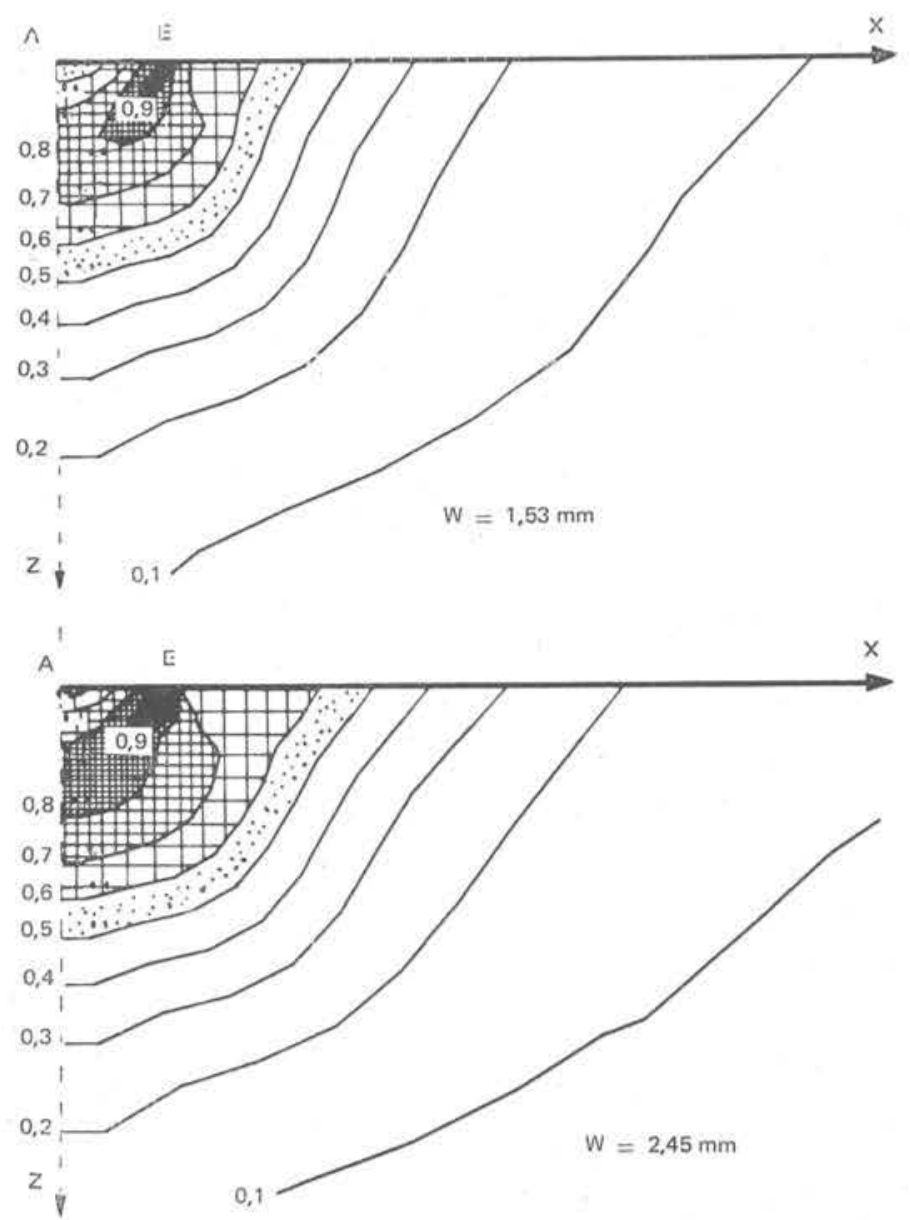

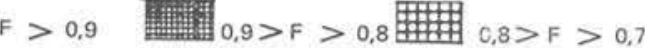

$\boxplus_{0,7>F>0,6} \square_{0,6>F}>0,5 \square-5>F$

Fig. 19. - Enfoncement de plaque de $2 \mathrm{~J} \mathrm{~cm}$ : évolution de $\mathbf{F}=\frac{\sigma_{1} / \sigma_{3}^{\prime}-1}{\operatorname{tg}^{2}(\pi / 4+\Phi / 2)}$ 
Fig. 20. - Translation horizontale d'un écran vertical vers l'extérieur d'un massif : maillage et géométrie.

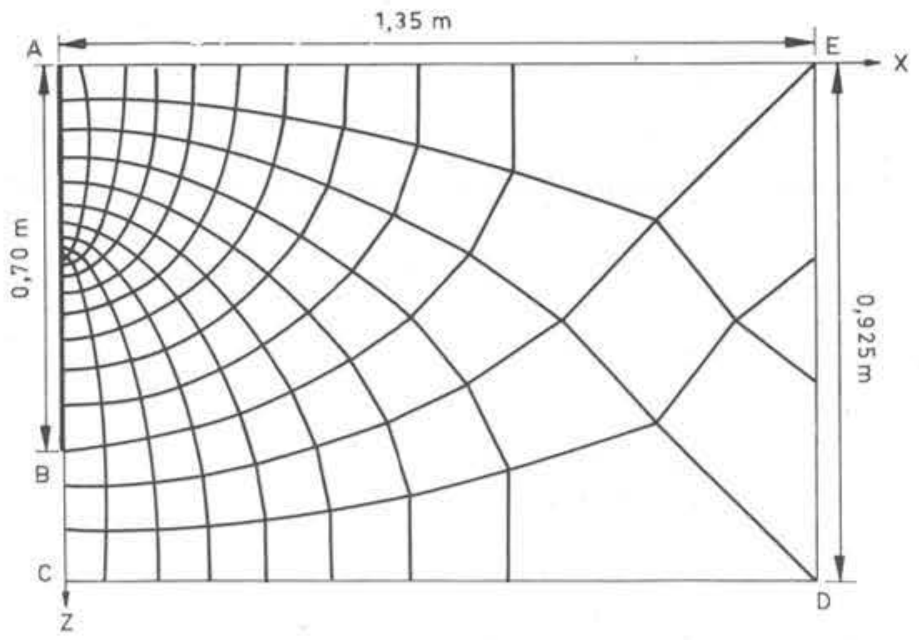

tout à fait les mêmes que celles du calcul. Le massif expérimental avait un prolongement au niveau de BC. En conséquence, les déplacements le long de BC n'étaient pas nuls. La différence entre calcul et expérience sera d'autant plus faible que l'on s'éloignera du point $\mathrm{B}$, base du mur.

\subsection{Résultats}

Les figures 21 et 22 présentent les comparaisons entre contraintes (respectivement normales et tangentielles, calculées et mesurées. Les états ne sont pas identiques, la mișe en place donnant un $\mathrm{K}_{o}$ très différent de la valeur 1 qu'implique l'initialisation isotrope du calcul. Aussi avons nous fait porter la comparaison sur des valeurs correspondant à des translations déjà importantes, l'état initial se trouvant alors en quelque sorte «effacé» par l'histoire subie par le matériau.

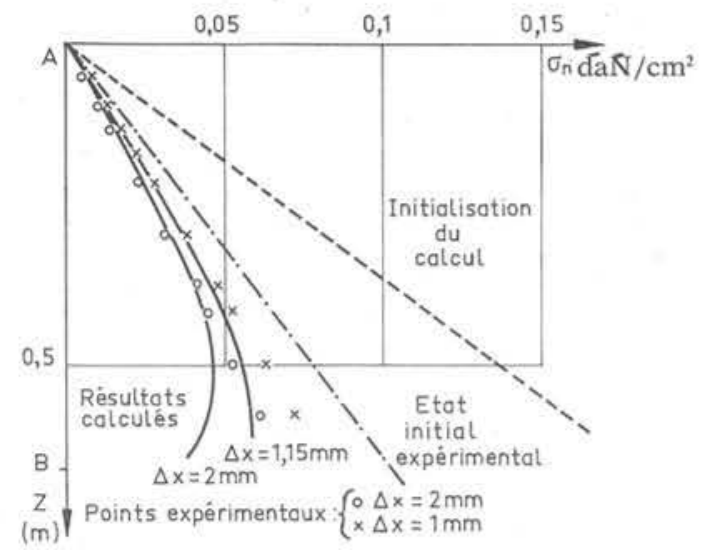

Fig. 21. - Translation horizontale d'un écran vertical vers l'extérieur d'un massif : comparaison des contraintes normales sur l'écran, calculées et mesurées.

Pour les valeurs de translation considérées, la concordance est bonne, pour les contraintes normales comme pour les contraintes tangentielles, sur les deux tiers supérieurs de l'écran. Pour le bas, la divergence s'explique par la remarque finale du paragraphe 3.51 .

La figure 23 montre l'évolution de $\mathrm{F}$ dans le massif au cours de la translation. On voit naître deux zones

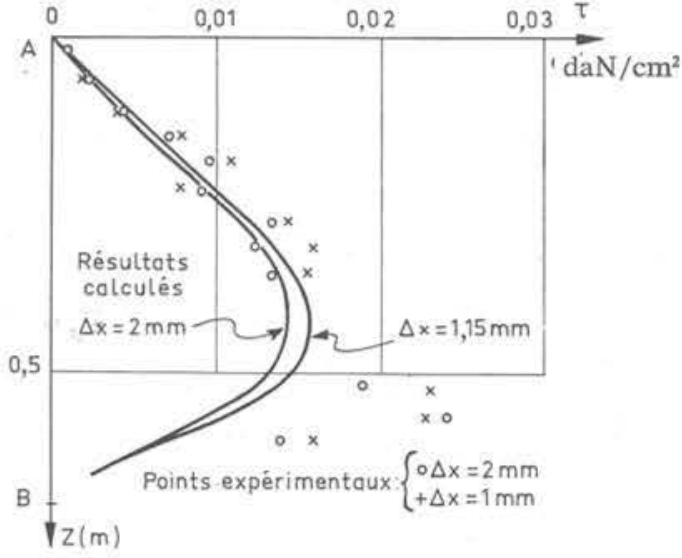

Fig. 22. - Translation horizontale d'un écran vertical vers l'extérieur d'un massif : comparaison des contraintes tangentielles sur l'écran, calculées et mesurées.

"plastiques», l'une au voisinage de la singularité (le point B pied de l'écran) l'autre le long de cet écran et partant du haut du massif. L'état du massif pour $\Delta x=-1.688 \mathrm{~mm}$, semble conduire à une rupture avec une seule ligne de discontinuité partant de la base de l'écran.

\subsection{Translation d'un écran vertical vers l'intérieur d'un massif}

\subsection{Définition et position du problème}

La géométrie et le maillage sont représentés sur la figure 24. Le massif mesure $60 \mathrm{~cm}$ de haut sur $135 \mathrm{~cm}$ de large, l'écran $\mathrm{AB}$ a une hauteur de $36 \mathrm{~cm}$ et il est supposé parfaitement rugueux. Les conditions aux limites sont les suivantes : les déplacements sur BCDE sont nuls; la contrainte sur EA est nulle ; sur $\mathrm{AB}$ les déplacements verticaux sont nuls et les déplacements horizontaux égaux (à $\Delta x$ ).

Le maillage comporte cent huit éléments ( 130 noeuds) et est réalisé par le même programme de maillage, afin que l'on ait de petites mailles le long de l'écran.

L'initialisation est identique à celle des problèmes précédents. Comme pour la translation vers l'extérieur 

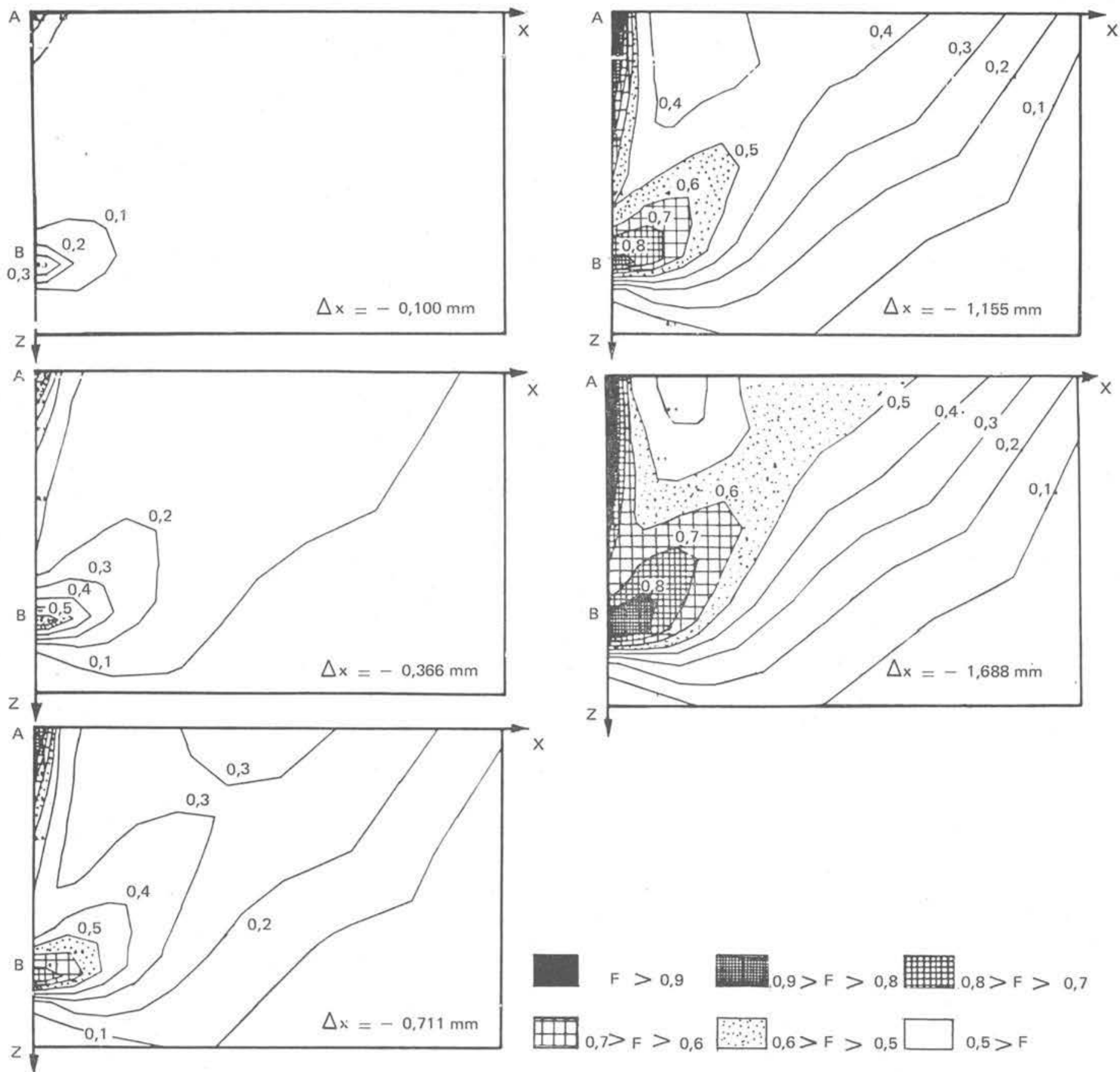

Fig. 23. - Translation horizontale d'un écrạ vertical vers l'extérieur d'un massif : évolution de $\mathbf{F}=\frac{\sigma_{1} / \sigma_{3}-1}{\operatorname{tg}^{2}(\pi / 4+\Phi / 2)}$

Fig. 24, - Translation horizontale d'un écran vertical vers l'intérieur d'un massif : maillage et géométrie.

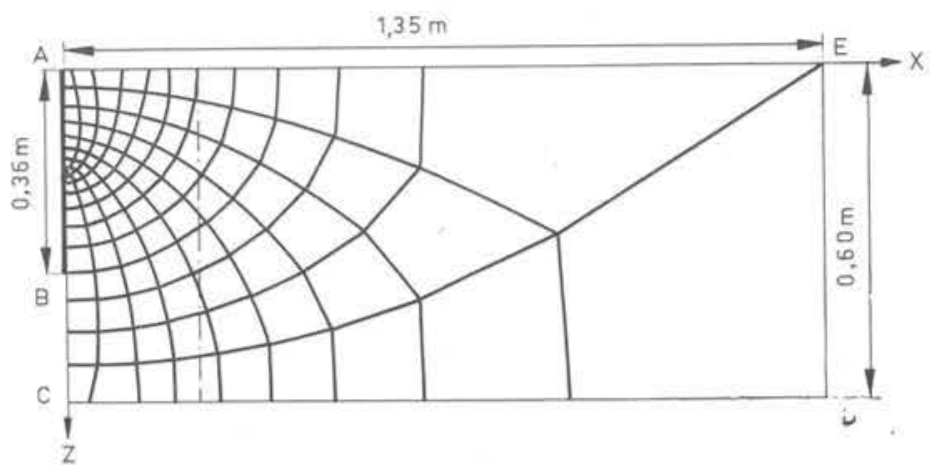




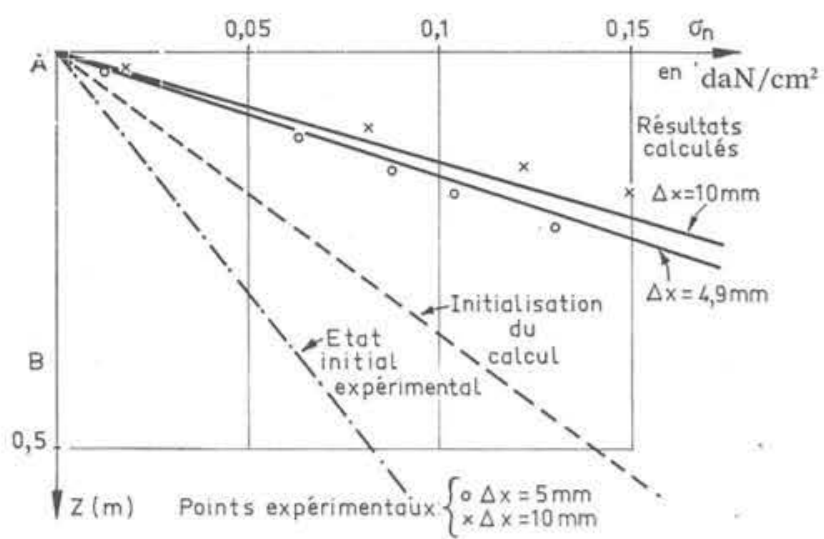

Fig. 25. - Translation horizontale d'un écran vertical vers l'intérieur d'un massif : comparaison des contraintes normales sur l'écran calculées et mesurées.

du massif, les conditions aux limites sur BC sont sûrement différentes des véritables conditions de l'expérience pour laquelle le massif se prolongeait de l'autre côté de l'écran, au niveau de BC.

La translation de l'écran $(1 \mathrm{~cm})$ est divisée en dix incréments variant en progression arithmétique.

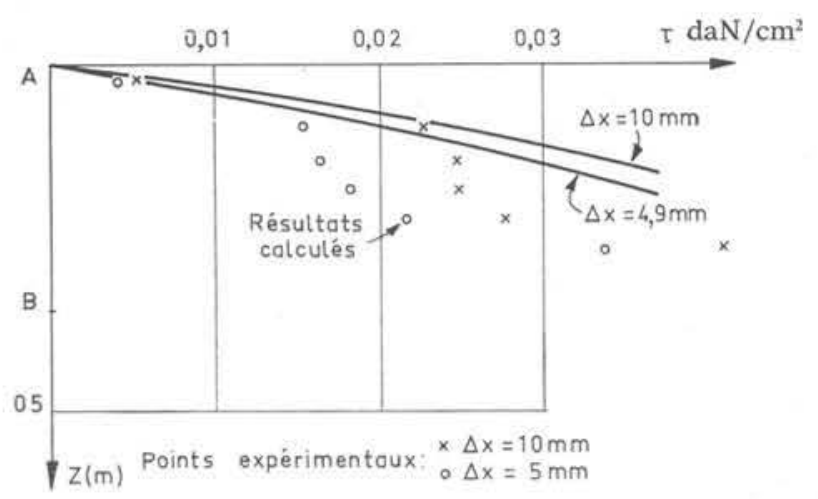

Fig. 26. - Translation horizontale d'un écran vertical vers l'intérieur d'un massif : comparaison des contraintes tangentielles sur l'écran, calculées et mesurées.

\subsection{Résultats}

Les figures 25 et 26 présentent la comparaison entre contraintes (respectivement normales et tangentielles) calculées et mesurées. Comme pour le calcul précédent, les états initiaux étant différents, nous avons fait porter
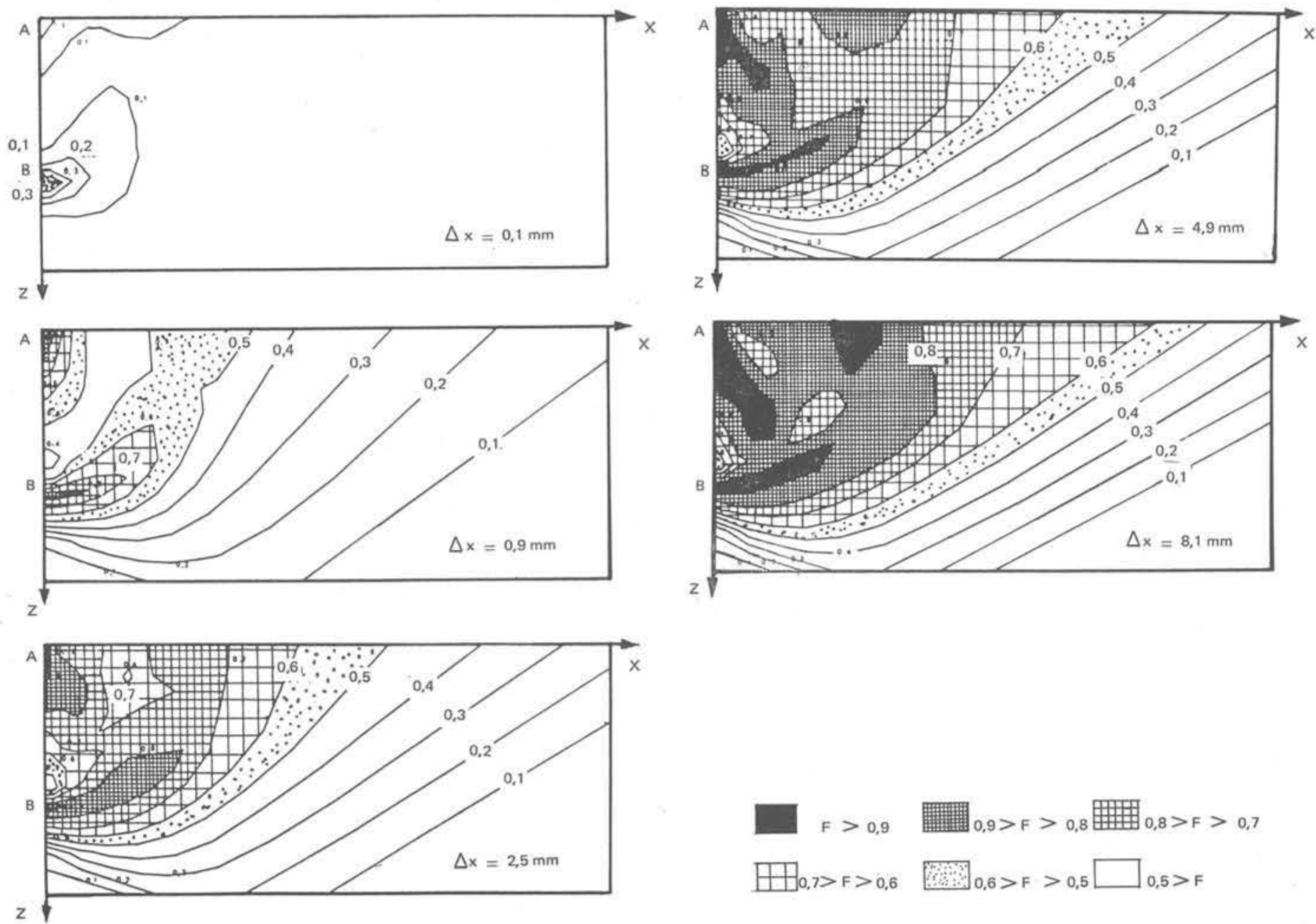

Fig. 27, - Translation horizontale d'un écran vertical vers l'intérieur d'un massif : évolution de $\mathbf{F}=\frac{\sigma_{1} / \sigma_{3}-1}{\operatorname{tg}^{2}(\pi / 4+\Phi / 2)}$ 
la comparaison sur des valeurs correspondant à des translations relativement grandes.

Comme dans le cas précédent, la concordance est bonne pour les deux tiers supérieurs de l'écran et pour les contraintes normales. Par contre, les contraintes tangentielles calculées et mesurées, diffèrent sensiblement, bien que l'ordre de grandeur soit respecté.

La figure 27 montre l'évolution de $\mathrm{F}$ dans le massif au cours de la translation. On voit naître deux zones "plastiques» de façon un peu analogue (bien que dissymétriques sans doute à cause de la pesanteur) à celle qui prennent naissance sur le bord d'une fondation superficielle.

L'état final du massif semble suggérer une rupture avec deux lignes de discontinuité dans le massif, l'une partant du bas de l'écran et l'autre du haut, ce qui diffère notablement du cas précédent. A ce propos, il convient de remarquer que nous retrouvons qualitativement, tant en poussée qu'en butée, les résultats expérimentaux obtenus sur la station de poussée-butée en vraie grandeur de St-Rémy-lès-Chevreuse (CEBTP).

\section{CONCLUSION}

Nous voudrions, pour terminer, résumer les caractéristiques essentielles de notre étude ainsi que les résultats que l'on peut en attendre dans l'avenir.

La loi incrémentale que nous proposons est tridimensionnelle; elle permet de décrire de manière continue le comportement d'un sable, depuis les plus faibles déformations, quasi-élastiques, jusqu'aux plus grandes, atteintes en plasticité parfaite. Elle est valable en " charge " comme en " décharge " (c'est-à-dire même si les sollicitations ne sont pas monotones) et rend compte du caractère irréversible des déformations d'un matériau pulvérulent. L'influence de l'histoire antérieure du matériau (chemin de sollicitation suivi antérieurement) est prise en compte. A l'heure actuelle, elle ne prend pas en compte l'effet du temps mais l'adjonction d'un terme visqueux est en cours (il ne pose d'ailleurs que peu de problèmes théoriques nouveaux). Par contre, nous ne pouvons pas décrire le comportement du sol sous sollicitation dynamique sans un développement important.

Sous sa forme simplifiée, intégrée dans des programmes de calcul numérique basé sur la méthode des éléments finis, elle permet de retrouver avec une bonne précision les résultats expérimentaux de problèmes très différents : résultats d'autant plus satisfaisants que les paramètres utilisés pour le calcul sont dans tous les cas déterminés à partir de quelques essais triaxiaux. Bien sûr, les calculs ne portent que sur des expériences de laboratoire mais nous voudrions poursuivre cette confrontation sur des cas réels très bien instrumentés et, pour lesquels, les hypothèses de calcul (calcul plan par exemple) sont assez bien satisfaites, de façon à ne pas induire d'erreur qui ne serait due ni à la loi ni à la méthode de calcul.

Dans un avenir proche, nous serons en mesure d'élargir le champ des problèmes traités, par la prise en compte dans les calculs de conditions aux limites plus réalistes (frottement sol-structure par exemple avec loi de Coulomb).

Dans un avenir plus lointain, nous pensons que cette loi, intégrée dans des programmes basés sur la méthode des éléments finis et écrite sous sa forme générale (matrice non symétrique, possibilités de sollicitations non monotones), devrait permettre de joindre en un même calcul les déterminations du tassement et de la force portante, même dans le cas de cycles de " charge "- " décharge ". 


\section{REFERENCES BIBLIOGRAPHIQUES}

[1] SALENCON. - « Théorie de la plasticité pour les applications à la mécanique des sols », Eyrolles, Paris (1974).

[2] ZIENKIEWICZ. - « La méthode des éléments finis », Ediscience, Paris (1973).

[3] DUCAN, CHANG. - « Non linear analysis of stress and strain in soils ». Jour. of Soil mechanics and foundations, division ASCE, vol. 96, no 5 (1970).

[4] FRANK. - « Etude théorique du comportement des pieux sous charge verticale, introduction de la dilatance ». Rapport de recherche $n^{\circ} 46$, Labo. des Ponts et Chaussées (juillet 1975).

[5] REYES and DEERE. - * Elastic-plastic analysis of underground openings by the finite element method ". $1^{\text {er }}$ Congrès international de mécanique des roches, II, 7.42, Lisbonne (1966).

[6] WHITMANN. - « Two dimensional analysis of stress and strain in soils ", Contract report $\mathrm{n}^{\circ}$ 3, 129 for U.S. Army Eng. WES, by dep. of MIT, Cambridge (1965).

[7] RICARD. - « Résolution des problèmes d'élastoplasticité par la méthode des éléments finis ». Laboratoire Central des Ponts et Chaussées (1973).

[8] ROSCOE, SCHOFIELD, WROTH. - « On the yielding of soils », Géotechnique, vol. VIII, no 1 (1958).

[9] DARVE. - " Contribution à la détermination de la loi rhéologique incrémentale des sols ", Thèse de Docteur-Ingénieur, Grenoble (1974).

[10] DARVE, LABANIEH. - " Loi rhéologique incrémentale des milieux pulvérulents ", Congrès Français de Mécanique, Toulouse (1975).
[11] LADE. - « The stress-strain and strength characteristics of cohesionless soils », Thesis of Doctor of Philosophy Berkeley (1972).

[12] LADE, DUNCAN. - « Cubical trixial tests on cohesionless soil ", Journal of the soil Mechanics and Foundations Division, ASCE, vol. 99, $\mathrm{n}^{\circ}$ SM 10 (oct. 1973).

[13] SCHNEEBELI. - «Une analogie mécanique pour les terres sans cohésion " C.R.A.S., tome 243 , p. 125 (1956).

[14] PIOLINE. - - Etude de quelques problèmes de soutènements en petites déformations ». Thèse de Docteur de Spécialité, Grenoble (1975).

[15] WACK. - « Mesure par radioactivité en mécanique des sols ", Thèse de doctorat ès Sciences, Grenoble (1968).

[16] TERRIEZ. - « Sur l'identification optimale de lois de comportement élastoplastiques isotropes ", Thèse de doctorat de spécialité, Grenoble (1974).

[17] CHAMBON. - «Application de la méthode des éléments finis et d'une loi rhéologique incrémentale aux calculs de mécanique des sols ", Thèse de docteur-ingénieur, Grenoble (1975).

[18] TERZAGHI, PECK. - " Mécanique des sols appliquée », Dunod (1965).

[19] TURPAIN, - " Contribution à l'étude de la répartition des contraintes sous une fondation plane rugueuse en milieu bidimensionnel pulvérulent ", Thèse de doctorat de spécialité, Grenoble (1963).

[20] STUTZ. - « Contribution à l'étude de la loi de déformation plastique des sols ", Thèse de doctorat de spécialité, Grenoble (1961). 

